\title{
1 CDK13 Mutations Drive Melanoma via Accumulation of Prematurely Terminated
}

\section{Transcripts}

3 Megan L. Insco ${ }^{1,2}$, Brian J. Abraham ${ }^{3}$, Sara J. Dubbury ${ }^{4,5}$, Sofia Dust ${ }^{6}$, Constance Wu ${ }^{1}$, Kevin Y.

4 Chen $^{1}$, David Liu ${ }^{2}$, Calvin G. Ludwig ${ }^{1}$, Stanislav Bellaousov $^{7}$, Tania Fabo ${ }^{1}$, Telmo Henriques ${ }^{8}$,

5 Karen Adelman ${ }^{8}$, Matthias Geyer ${ }^{6}$, Phillip A. Sharp ${ }^{4,5}$, Richard A. Young ${ }^{5,9}$, Paul L. Boutz ${ }^{7,10,11}$,

6 and Leonard I. Zon ${ }^{1, *}$

$8{ }^{1}$ Stem Cell Program and Division of Hematology/Oncology, Boston Children's Hospital,

9 Howard Hughes Medical Institute, Boston, MA, USA. ${ }^{2}$ Department of Medical Oncology, Dana-

10 Farber Cancer Institute, Boston, MA, USA. ${ }^{3}$ Department of Computational Biology, St. Jude

11 Children's Research Hospital, Memphis, TN, USA. ${ }^{4}$ The David H. Koch Institute for Integrative

12 Cancer Research, Massachusetts Institute of Technology, Cambridge, MA, USA. ${ }^{5}$ Department of

13 Biology, Massachusetts Institute of Technology, Cambridge, MA, USA. ${ }^{6}$ Institute of Structural

14 Biology, University of Bonn, Bonn, DE. ${ }^{7}$ University of Rochester School of Medicine and

15 Dentistry, Rochester, NY, USA. ${ }^{8}$ Department of Biological Chemistry and Molecular

16 Pharmacology, Harvard Medical School, Boston, MA, USA. ${ }^{9}$ Whitehead Institute for

17 Biomedical Research, Massachusetts Institute of Technology, Cambridge, MA, USA. ${ }^{10}$ Center

18 for RNA Biology, University of Rochester, Rochester, NY, USA. ${ }^{11}$ Center for Biomedical

19 Informatics, University of Rochester, Rochester, NY, USA.

$21 *$ Corresponding author: leonard.zon@enders.tch.harvard.edu

\section{Abstract}


24 Transcriptional Cyclin Dependent Kinases modulate RNA Polymerase II function to impact gene

25 expression. Here, we show that $C D K 13$ is mutated in $4 \%$ of patient melanomas and mutation or

26 downregulation is associated with poor overall survival. Mutant CDK13 lacks kinase activity and

27 overexpression in zebrafish leads to accelerated melanoma. CDK13 mutant fish and human

28 melanomas accumulate prematurely terminated RNAs that are translated into truncated proteins.

29 CDK13 binds to and regulates the phosphorylation of $\mathrm{ZC} 3 \mathrm{H} 14$, a member of the $\underline{\text { Poly }} \underline{\mathrm{A}}$

30 exosome Targeting (PAXT) RNA degradation complex. ZC3H14 phosphorylation recruits the

31 PAXT complex to degrade prematurely terminated polyadenylated transcripts in the nucleus. In

32 the presence of mutant $\mathrm{CDK} 13, \mathrm{ZC} 3 \mathrm{H} 14$ phosphorylation is compromised and consequently fails

33 to recruit the PAXT complex, leading to truncated transcript stabilization. This work establishes

34 a role for CDK13 and the PAXT nuclear RNA degradation complex in cancer and has prognostic

35 significance for melanoma patients with mutated or downregulated CDK13.

Transcriptional Cyclin Dependent Kinases (CDKs) are a phylogenetically-related family of

38 kinases that are activated by a cyclin binding partner ${ }^{1}$ and have roles in transcriptional

39 subprocesses including initiation, elongation, and recruitment of complexes engaged in

40 cotranscriptional RNA processing ${ }^{2-5}$. Transcriptional CDKs are being explored as drug targets

41 for many difficult-to-treat cancers ${ }^{6-9}$. Additionally, mutations affecting one of the transcriptional

42 CDKs, CDK13, have recently been found to cause syndromic developmental disorders that affect

43 neural-crest derived tissues ${ }^{10,11}$ including heart and craniofacial developmental anomalies.

44 However, the role of CDK13 in transcription and RNA processing remains poorly understood

45 and no functional role for CDK13 has been defined in cancer.

46 RNA polymerase II (RNAPII), the enzyme that transcribes pre-messenger RNA from 
47 protein-coding genes, is exquisitely regulated at multiple steps to ensure transcriptional

48 initiation, elongation, and termination are precisely executed. During active elongation, a rapid

49 cascade of a vast number of RNA processing steps takes place ${ }^{12}$. Given this complexity, RNA

50 processing errors can occur, resulting in defective transcripts that must be degraded by the

51 nuclear exosome in order to prevent their translation into truncated proteins ${ }^{15,16}$.

52 The nuclear exosome degrades aberrant or unstable nuclear RNAs. Three different human

53 adaptor complexes have been discovered to date, each of which associates with and directs a

54 specific class of nuclear RNAs for exosomal degradation. All three adaptor complexes include

55 the MTR4 helicase, paired with proteins that recognize distinct RNA species. The TRAMP

56 complex targets aberrant nucleolar ribosomal RNAs while the NEXT complex targets PROMoter

57 uPstream Transcripts (PROMPTs) and enhancer RNAs for destruction ${ }^{13}$. The recently reported

58 Poly스 tail exosome $\underline{\text { Targeting }}$ (PAXT) complex degrades prematurely terminated

59 polyadenylated RNAs and requires the adaptor protein $\mathrm{ZFC} 3 \mathrm{H} 1^{14,15}$. It is unknown how PAXT-

60 mediated RNA degradation is regulated.

61 In this study, we show that mutations in $C D K 13$ occur in melanoma and promote tumor

62 formation and growth. The accelerated tumor progression in melanomas is dependent on Cyclin

63 T1 (CCNT1) rather than the canonical CDK13 cyclin partner, Cyclin K (CCNK). When CDK13

64 is mutated, ZC3H14 phosphorylation is compromised, and consequently the nuclear RNA

65 exosome fails to be recruited to prematurely terminated polyadenylated RNAs. Failure to recruit

66 the nuclear RNA exosome to prematurely truncated transcripts results in accumulation of

67 aberrant RNAs and the production of truncated proteins in melanoma. This work uncovers a role

68 for the PAXT nuclear RNA exosome in human cancer and defines its regulation by CDK13. 


\section{Results}

\section{CDK13 is a melanoma tumor suppressor.}

72 We examined the transcriptional CDK loci in melanoma patient samples and found CDK13 is

73 mutated in $4 \%(22 / 520$ cases $)$ of cutaneous melanomas, with 9 distinct missense mutations

74 occurring in the kinase domain, and 7 of these mutations near the ATP-binding site (Figure 1A).

75 Surprisingly, many of the same amino acid changes were found in CDK13-associated

76 developmental syndromes ${ }^{10}$, suggesting a specific activity of these mutations. Survival analysis

77 of human TCGA data revealed that $C D K 13$ downregulation $(z$ score $\leq-1.0)$ or mutation

78 (CDK13-altered) correlated with decreased overall survival [median CDK13-altered survival of

48.8 months vs. remaining case survival of 102 months ${ }^{16,17}$ (Figure 1B)]. Patients initially staged

80 with 0/1/2 melanoma that had $C D K 13$-alterations exhibited reduced overall survival compared to

81 remaining stage 1/2 melanoma patients (Figure S1A). CDK13 melanoma mutations near the

82 ATP-binding site were plotted onto the crystal structure (Figure $1 \mathrm{C}$ ) ${ }^{18}$ and $6 / 7$ of these mutations

83 were predicted to eliminate kinase activity by disruption of T-loop activation (R860Q or P869S),

84 alteration of the substrate binding pocket (W878L), or disruption of the kinase domain structure

85 (P881L, P893L, and I843N). In vitro, CDK13 ${ }^{\mathrm{WT}}$ activated by the typical cyclin partner, CCNK,

86 had robust kinase activity while the R860Q, W878L, and K734R mutants failed to phosphorylate

87 full-length RNAPII C-terminal domain (CTD) and a second substrate (Figure 1D, S1B). As a

88 control, we included the catalytically-dead CDK13 ${ }^{\mathrm{K} 734 \mathrm{R}}$ mutation, which replaces the lysine

89 required for catalysis of all known kinases. In summary, these data suggest that $C D K 13$ is

90 mutated in melanoma, mutations abrogate CDK13 kinase activity, and mutation or

91 downregulation of $C D K 13$ in patient melanomas is associated with decreased overall survival.

92 To examine $c d k 13$ loss in melanoma, we used a $B R A F^{V 600 E}$; p53-/-; mitfa-/- zebrafish

93 melanoma model ${ }^{19}$ in which Cas 9 was expressed in melanocytes using the mitfa promoter and 
94 either a $c d k 13$ gRNA or a control gRNA was ubiquitously expressed. Zebrafish lacking $c d k 13$

95 showed significantly decreased numbers of melanocytes as compared with zebrafish expressing a

96 control gRNA, indicating that $c d k 13$ is required for normal melanocyte development (Figure 1E,

$971 \mathrm{~F}, \mathrm{~S} 1 \mathrm{D}$ vs. S1C). Overexpression of human wild-type $C D K 13\left(C D K 13^{W T}\right)$ resulted in fewer

98 melanocytes during development (Figure 1E, 1F, S1E). Injection of a $c d k 13$ gRNA/Cas9-

99 expressing vector and human $C D K 13^{W T}$ rescued melanocyte numbers in the melanoma model

100 (Figure 1E, 1F, S1F). These data show that tightly-controlled levels of $c d k 13$ are required for

101 melanocyte development and that human $C D K 13$ can complement the loss of the zebrafish gene.

102 Rarely, melanomas arose in zebrafish with $c d k 13$ melanocyte-specific CRISPR deletion, and

103 these tumors appeared to grow faster than melanomas with intact $c d k 13$. Phospho-histone 3

104 (PH3) immunohistochemical (IHC) staining of melanomas with $c d k 13$ gRNA and a control

105 gRNA revealed that $c d k 13$ CRISPR-deleted tumors were significantly more proliferative than

106 melanomas with retained $c d k 13$ (Figure 1G, S1H). PCR across the CRISPR cut site confirmed

107 frameshift indels, and more in-frame indels were observed in $c d k 13$ gRNA compared to control

108 gRNA melanomas (Figure S1G). These data suggest that melanocytes with loss of $c d k 13$ may be

109 selected against during development, but surviving melanocytes give rise to highly proliferative

110 melanomas.

111 To test whether CDK13 melanoma-associated mutations cause more aggressive melanoma

112 in vivo, mutant CDK13 was expressed in melanocytes in a BRAFV600E; p53-/-; mitfa-/-

113 zebrafish melanoma model ${ }^{20,21}$. Control melanocyte-specific EGFP expression resulted in

114 expected mosaic zebrafish stripes, whereas human CDK13 ${ }^{\mathrm{WT}}$ expression resulted in dramatically

115 fewer melanocytes at 9wpf (Figure 1H, S1I). In contrast, melanocyte-specific overexpression of

$116 \mathrm{CDK}^{\mathrm{R} 860 \mathrm{Q}}, \mathrm{CDK} 13^{\mathrm{P} 869 \mathrm{~S}}, \mathrm{CDK} 13^{\mathrm{W} 878 \mathrm{~L}}, \mathrm{CDK} 13^{\mathrm{P} 881 \mathrm{~L}}$, and CDK13 ${ }^{\mathrm{P} 893 \mathrm{~L}}$ caused the appearance of 
117 black patches at 9wpf (Figure 1H, S1I) and expedited tumor onset (Figure 1I, S1J).

118 Overexpression of the catalytically-dead CDK13 ${ }^{\mathrm{K} 734 \mathrm{R}}$ mutant also caused black patches at $9 \mathrm{wpf}$

119 and expedited tumor onset (Figure 1H, 1I, S1I). CDK13 W878L- and P893L-expressing

120 melanomas had more PH3-positive cells than control EGFP melanomas by IHC (Figure 1J,

$121 \mathrm{~S} 1 \mathrm{~K})$. Because all CDK13 mutations promoted melanoma to a similar degree, we have used them

122 interchangeably in most assays, and refer to them collectively hereafter as $C D K 13^{\text {mel }}$. Since both

123 CRISPR-mediated deletion and CDK13 ${ }^{\text {mel }}$ expression in zebrafish caused more proliferative

124 melanomas, our data suggest that $\mathrm{CDK} 13^{\text {mel }}$ mutations act through a dominant negative

125 mechanism.

$126 \quad$ As CDK $13^{\mathrm{mel}}$ acts via a dominant negative mechanism and no CDK13 ${ }^{\text {mel }}$ mutations are

127 observed in the Cyclin binding domain, we hypothesized that Cyclin binding is required for

128 CDK13 ${ }^{\text {mel }}$ dominant negative activity. To test whether the canonical CDK13 Cyclin,

$129 \mathrm{CCNK}^{18,22,23}$, or the related CCNT1 are required for CDK13 ${ }^{\text {mel }}$ dominant negative

130 melanomagenesis in vivo, we co-injected vectors that express melanocyte-specific CDK13 ${ }^{\text {mel }}$ and

131 melanocyte-specific CRISPR of $c c n K$ or $c c n T 1$ in the $m i t f a^{-/} ; B R A F ; p 53^{-/}$zebrafish melanoma

132 model. $c c n K$ melanocyte-specific CRISPR expedited melanoma in the presence of CDK13 ${ }^{\mathrm{W} 878 \mathrm{~L}}$,

133 but not alone (Figure S1L-M). ccnT1 melanocyte-specific CRISPR suppressed CDK13 ${ }^{\text {W878L }}$ and

$134 \mathrm{CDK}^{\mathrm{R} 860 \mathrm{Q}}$ oncogenesis but had no effect by itself (Figure $\left.1 \mathrm{~K}-\mathrm{L}, \mathrm{S} 1 \mathrm{O}\right)$. PCR across the

135 CRISPR cut site confirmed indels directed by the $c c n T 1, c c n K$, and control gRNAs (Figure

$136 \mathrm{~S} 1 \mathrm{~N})$. These data indicate that $\mathrm{CDK} 13^{\mathrm{mel}}$ oncogenesis requires CCNT1 for its dominant negative

137 oncogenic activity.

138 Mutant CDK13 binds chromatin in a dominant negative manner. 
140 CTD (Figure 1D), chromatin immunoprecipitation sequencing (ChIP-seq) was used to determine

141 the localization of CDK13 ${ }^{\text {mel }}$ on melanoma chromatin. ChIP-seq was performed on

142 approximately equal cell numbers gathered from EGFP and CDK13 ${ }^{\mathrm{W} 878 \mathrm{~L}}$-expressing zebrafish

143 melanomas (Figure 2A). EGFP-expressing melanomas were used as the ChIP-seq control since

144 zebrafish expressing CDK13 ${ }^{\mathrm{WT}}$ had very few melanocytes and rarely developed melanoma.

145 ChIP-seq from EGFP melanomas showed zebrafish Cdk13 was especially enriched at

146 transcriptional start sites (TSSs) (Figure 2B, left, green). CDK13 ${ }^{\mathrm{W} 878 \mathrm{~L}}$ overexpression in

147 melanoma resulted in increased CDK13 signal at TSSs (Figure 2B, left, black). CDK13 ${ }^{\mathrm{W} 878 \mathrm{~L}}$

148 bound to genes with Cdk13 ${ }^{\mathrm{WT}}$ occupancy in control melanomas and the amount of CDK13 ${ }^{\mathrm{W} 878 \mathrm{~L}}$

149 bound correlated with the amount of $\mathrm{Cdk} 13^{\mathrm{WT}}$ bound in control melanomas (Figure 2C).

150 To determine whether mutant CDK13 also bound chromatin in human melanoma cells,

151 CLOVER fluorescent protein, CDK13 ${ }^{\mathrm{WT}}, \mathrm{CDK} 13^{\mathrm{W} 878 \mathrm{~L}}$, or CDK13 ${ }^{\mathrm{R} 860 \mathrm{Q}}$ were expressed in

152 human A375 melanoma cells (Figure 2E). Cells expressing either CDK13 ${ }^{\text {mel }}$ protein grew more

153 quickly than cells expressing CDK13 ${ }^{\mathrm{WT}}$ or CLOVER (Figure 2F, Figure S2A). CDK13 ${ }^{\mathrm{R} 860 \mathrm{Q}}$ also

154 bound chromatin loci previously bound by CDK13 ${ }^{\mathrm{WT}}$, similar to the findings in zebrafish

155 melanomas (Figure 2G-H). Because CDK13 ${ }^{\text {mel }}$ binds chromatin at sites normally bound by

$156 \mathrm{CDK} 13^{\mathrm{WT}}, \mathrm{CDK} 13^{\mathrm{mel}}$ is positioned to interfere with $\mathrm{CDK} 13^{\mathrm{WT}}$ kinase activity and thus act in a

157 dominant negative manner in zebrafish and human melanoma cells.

As CDK13 ${ }^{\text {mel }}$ bound chromatin, we next sought to determine if CDK13 ${ }^{\text {mel }}$ disrupted RNAPII

159 occupancy. ChIP-seq was performed with antibodies that recognize either RNAPII (anti-

160 8WG16), or RNAPII phosphorylated at the CTD Serine-2 position (anti-Ser2p), a modification

161 of elongating RNAPII involved in the recruitment of RNA processing machinery 12,25,26.

162 Zebrafish melanomas expressing CDK13 ${ }^{\text {W878L }}$ exhibited perturbed RNAPII as compared to 
163 control melanomas, with a notable decrease in TSS-proximal RNAPII and a slight increase gene

164 body RNAPII (Figure 2D). Ser2p RNAPII occupancy was also perturbed; genes with the highest

165 increase in CDK13 ${ }^{\text {mel }}$ binding had increased Ser2p (Figure S2B, left). When the Ser2p RNAPII

166 signal was normalized to total RNAPII, a dramatic increase in Ser2P RNAPII was observed at

167 the promotor-proximal region (Figure S2C, left). Promoter-proximal enrichment of Ser2P

168 RNAPII has been reported for conditions causing slowed RNAPII ${ }^{27}$. Human melanoma cell

169 CDK13 ${ }^{\mathrm{R} 860 \mathrm{Q}}$ expression caused similar perturbations in RNAPII occupancy [Figure 2I, S2B

170 (right), S2C (right)]. To ascertain whether CDK13 ${ }^{\text {mel }}$ expression impacts transcription based on

171 gene expression level or gene length, we compared the RNAPII and Ser2P ChIP signals in gene

172 subsets partitioned by gene expression level or gene length. More CDK13 ${ }^{\text {mel }}$ was bound and

173 RNAPII was more perturbed on highly expressed genes (Figure S2D-E), however there was no

174 correlation with gene length (S2F-G). These data show CDK13 ${ }^{\text {mel }}$ expression perturbed RNAPII

175 occupancy on highly expressed genes, suggesting that CDK13 normally acts via a mechanism

176 important on highly expressed genes.

177 Notably, changes in the distribution of the Ser2p signal upon CDK13 ${ }^{\text {mel }}$ expression differed

178 distinctly from those observed upon loss of a close paralog, CDK12. In cells lacking CDK12,

179 Ser2p was strongly decreased across the gene body despite an increase in total RNAPII ${ }^{28}$. These

180 data also suggest that $\mathrm{CDK} 13^{\mathrm{mel}}$ promotes oncogenesis via a mechanism that is distinct from

181 CDK12.

182 Mutant CDK13 causes accumulation of prematurely-terminated RNAs.

183 To determine the transcriptional impact of $\mathrm{CDK} 13^{\mathrm{mel}}$ in zebrafish melanomas, RNA

184 sequencing (RNA-seq) was conducted on BRAF; p53-/-; mitfa-/- zebrafish melanomas

185 expressing $\operatorname{EGFP}(\mathrm{n}=4)$ or mutant $C D K 13(R 860 Q, \mathrm{n}=5 ; K 734 R, \mathrm{n}=5$, Figure S3A). Differential

186 expression reassuringly showed the most differentially expressed gene in the zebrafish 
187 melanomas was human $C D K 13^{\text {mel }} .158$ downregulated and 53 upregulated genes with a $q$ value

$188<0.05$ were identified. Panther GO-Slim Biologic Process pathway analysis of upregulated genes

189 showed no pathway enrichment, while downregulated genes were enriched for 1) positive

190 regulation of GTPase activity $(\mathrm{q}=1.63 \times 10-7), 2)$ cellular response to tumor necrosis factor (TNF)

191 (q=1.64x10-7), and 3) granulocyte chemotaxis $(\mathrm{q}=1.61 \times 10-7)$.

192 As CDK13 is phylogenetically related to known transcriptional kinases CDK9 and CDK12,

193 and CDK13 ${ }^{\text {mel }}$ expression caused a perturbation in RNAPII occupancy, we tested for a global

194 RNA defect by quantifying differential exon expression. RNA differential expression analysis of

195 first (F), alternative first (AF), internal (I), last (L), or alternative last (AL) exons in each gene

196 were quantified genome-wide in zebrafish melanomas. Many genes exhibited increased read

197 coverage in the first exon as compared to the last exon in CDK13 ${ }^{\text {mel }}$ expressing as compared with

198 control melanomas ( $\mathrm{p}=2.2 \times 10^{-16}$, two-sided Wilcoxon rank sum test) (Figure $\left.3 \mathrm{~A}\right)$. These data

199 show that prematurely terminated transcripts containing the 5 ' but not the 3 ' end of the gene are

200 present in CDK13 ${ }^{\text {mel }}$ zebrafish melanomas.

201 To test whether $C D K 13^{\text {mel }}$ mutations have a similar effect on RNAs in patient melanomas,

202 we analyzed polyA-selected RNAseq data from TCGA data. Melanomas with kinase domain

203 mutations $(n=3)$ were compared to matched wild-type controls $(n=5)$. Similar to zebrafish

204 melanomas, increased read coverage in the first exon versus the last exon was observed in

$205 C D K 13^{m e l}$ as compared to $C D K 13^{W T}$ patients $\left(\mathrm{p}=2.2 \times 10^{-16}\right.$, two-sided Wilcoxon rank sum test)

206 (Figure 3B). One example gene that was significantly affected in patient samples was TRIM25

207 (Figure 3D). The evidence for prematurely terminated RNAs was of greater magnitude in polyA-

208 selected RNA-seq, consistent with the prematurely terminated transcripts being polyadenylated.

209 These data demonstrate that CDK13 ${ }^{\text {mel }}$ expression in both zebrafish and human melanomas 
210 results in the accumulation of prematurely terminated polyadenylated transcripts.

211 To determine whether genetic deletion of $C D K 13$ also results in accumulation of

212 prematurely terminated transcripts, we generated mouse embryonic stem cells (mESCs) that

213 were $C d k 13$-/- and carried a doxycycline-inducible $C d k 13$ rescue transgene. After removal of

214 doxycycline for 0,48 , or 72 hours, polyA-selected RNA was subjected to stranded sequencing

215 (Figure S3B-E). Similar to zebrafish and patient melanomas expressing CDK13 ${ }^{\text {mel }}$, increased

216 first exon coverage and decreased last exon coverage was observed at 48 hours as compared to 0

217 hours in Cdk13-depleted mESCs. Cdk13-depleted mESCs exhibited an additional increase in the

218 ratio of first to last exon coverage after 72 hours (Figure 3C). This data further supports the

219 hypothesis that Cdk13 is required to prevent accumulation of prematurely terminated

220 polyadenylated transcripts.

221 Notably, in contrast to the increase in distinctly positioned intronic polyadenylation events

222 observed upon Cdk12 depletion ${ }^{28}$, we were unable to detect cleavage and polyadenylation at

223 specific sites within gene bodies. We developed an algorithm to quantify sequencing reads with

224 non-genomically encoded polyA tails that map to the distal polyA sites of genes. Using this

225 algorithm, we observed a significant decrease in the ratio of transcripts from highly expressed

226 polyadenylated genes at the distal polyA site (corresponding to the full-length mRNA) to total

227 transcript number in Cdk13-depleted mESCs (Figure 3E). Cdk13 depletion causes RNAs

228 terminating upstream of the normal polyA site to be either generated more frequently or

229 degraded more slowly. As a consequence, the pool of cellular transcripts contains

230 proportionately fewer full-length mRNAs.

231 Mutant CDK13 causes accumulation of truncated proteins.

232 Prematurely terminated transcripts are typically degraded by the nuclear exosome, which 
233 prevents them from being exported and translated. To determine whether the prematurely

234 terminated transcripts we observed in CDK13 ${ }^{\mathrm{mel}}$ zebrafish melanomas are translated, we used

235 tandem mass spectrometry to analyze global protein expression in melanomas isolated from age-

236 matched $B R A F^{V 600 E} ;$ p53-/-; mitfa-/- zebrafish with melanocyte-specific expression of CDK13 ${ }^{\text {mel }}$

$237(n=3)$ or EGFP ( $n=3)$. To identify consistent changes across replicates, peptides measurements

238 with a t-test result of $\mathrm{p}<0.1$ were mapped. The $\log _{2}$-fold change in peptide measurements

239 between CDK13 ${ }^{\text {mel }} /$ EGFP were binned and plotted along protein length to create a metaplot

240 (Figure 4A, $\mathrm{r}=-0.5349, \mathrm{p}$ value=0.0001). The negative slope in the metaplot is consistent with

241 an increase in short proteins arising from the translation of prematurely terminated

242 polyadenylated transcripts. These data suggest that rather than being degraded by the nuclear

243 exosome, prematurely terminated transcripts accumulate and are translated into truncated

244 proteins in CDK13 ${ }^{\text {mel }}$ expressing cells.

245 To identify specific proteins that are most impacted by CDK13 ${ }^{\text {mel }}$, two methods were used.

246 First, we assessed proteins as shown in Figure 4A that had $>1$ measurement and metaplot slope

$247<-1$, which identified 263 truncated proteins. Second, using all data, proteins with $>3$ peptide

248 measurements were tested for a negative slope $\left(\log ^{2} \mathrm{CDK} 13^{\mathrm{mel}} / \mathrm{EGFP}\right.$, F-test $\left.\mathrm{p}<0.05\right)$. This more

249 restrictive analysis identified 103 candidates (Figure S4), 56 of which were distinct from the first

250 group. Of these 103 candidate proteins, the most significantly enriched KEGG Pathways were

251 metabolic ribosome (FDR 2.01e-07) and carbon metabolism (3.01 e-05). Several of the truncated

252 proteins we identified are predicted to lose carboxy-terminal enzymatic domains, including Ilk,

253 Crk, Ikbkb, and Cdk15 (Figure 4B-E). Ilk, Crk, and Ikbkb have published evidence of dominant

254 negative activity upon carboxy-terminal loss ${ }^{29-31}$. Individual impacted proteins showed an

255 increase in truncated species at the expense of full-length proteins in CDK13 ${ }^{\text {mel }}$ zebrafish 
melanomas. These data indicate that $\mathrm{CDK} 13^{\text {mel }}$ expressing zebrafish melanomas translate

257 prematurely terminated transcripts to produce truncated proteins, which we hypothesize cause

$258 \mathrm{CDK}_{13}{ }^{\mathrm{mel}}$ oncogenesis via tumor suppressor loss and/or expression of dominant negative protein

259 truncations.

260 To determine whether truncated protein production correlated with CDK13 ${ }^{\text {mel }}$ binding at the

261 gene locus, we overlaid the 319 truncated proteins with the top quintile of genes with enriched

262 CDK13 ${ }^{\text {mel }}$ binding as determined by ChIP-seq. 133/319 truncated proteins (42\%) were within the

263 top quintile of CDK13 ${ }^{\text {mel }}$ enrichment. As the ChIP-seq and tandem mass spectrometry

264 experiments were done on different cohorts of primary zebrafish melanomas, and given the

265 detection limits of mass spectrometry, the overlap in this restrictive analysis suggests that genes

266 with high levels of CDK13 ${ }^{\text {mel }}$ chromatin binding are more likely to accumulate prematurely

267 terminated RNAs which are translated into truncated proteins than would be expected by chance

$268(\mathrm{p}<0.0001$, Fisher's exact test $)$.

269 Mutant CDK13 disrupts the polyA RNA exosome.

270 To elucidate how CDK13 ${ }^{\text {mel }}$ expression results in the accumulation of truncated RNAs in

271 human melanoma cells, V5-tagged $\operatorname{CDK}^{\mathrm{WT}}(\mathrm{n}=3), \mathrm{CDK}^{\mathrm{W}}{ }^{\mathrm{W} 878 \mathrm{~L}}(\mathrm{n}=2)$, or control fluorescent

272 protein CLOVER ( $\mathrm{n}=3$ ) were immunoprecipitated from nuclear extracts and co-

273 immunoprecipitating proteins were identified by mass spectrometry (IP-MS) (Figure 5A). In the

274 CDK13 ${ }^{\mathrm{WT}}$ IP, of the 11 co-precipitating proteins identified (Figure 5C), 9 are RNA binding

275 proteins $\left(q=3.04 \times 10^{-5}\right)$ and 3 are involved in negative regulation of polyadenylated RNA

$276\left(q=2.73 \times 10^{-4}\right)^{32,33}$. We found both CDK13 ${ }^{\mathrm{WT}}$ and CDK13 ${ }^{\mathrm{W} 878 \mathrm{~L}}$ bound to CCNT1, again

277 implicating CCNT1 as the required cyclin-binding partners for CDK13 in human melanoma cells

278 as our functional data suggested (Figure 2J-L); surprisingly, the canonical cyclin binding partner 
of CDK13, CCNK, was not detected above background ${ }^{18,22,34}$. This unbiased approach indicated that the most significant interacting partners of CDK13 are CCNT1 and multiple factors involved in RNA processing.

In the $\mathrm{CDK} 13^{\mathrm{WT}} \mathrm{IP}$, the most abundantly identified interacting protein was $\mathrm{ZC} 3 \mathrm{H} 14$, a zinc finger protein implicated in negative regulation of polyA tail length ${ }^{35,36}$. ZC3H14 was also

284 recently identified as part of the Poly스 tail eX $\underline{\text { Xosome }}$ Targeting (PAXT) complex ${ }^{14}$ which is

285 responsible for targeting prematurely terminated polyadenylated transcripts to the nuclear 286 exosome for degradation ${ }^{15}$. Of the mutations observed in melanoma patients, $C D K 13^{W 878 L}$ was

287 chosen because it changes an amino acid in the substrate binding groove, which we hypothesized 288 would disrupt substrate binding (Figure 5B). In the CDK13 ${ }^{\mathrm{W} 878 \mathrm{~L}} \mathrm{IP}$, the ZC3H14 interaction was 289 strongly disrupted $(q=0.0195)$ while all other protein interactors were unaffected (Figure 5C).

290 These data suggest that ZC3H14 binds CDK13 in the substrate binding groove and could be a 291 CDK13 substrate.

To identify ZC3H14 binding partners in CDK13 ${ }^{\text {mel }}$ cells, the nuclear isoform of ZC3H14 293 and a control protein were tagged and transiently expressed in CDK13 ${ }^{\text {mel }}$ and CDK13 ${ }^{\mathrm{WT}}$ human 294 melanoma cells (Figure 5D). By IP-MS, we found that the three most abundant binding partners 295 for ZC3H14 in CDK13 ${ }^{\mathrm{WT}}$ cells were THOC2, ZFC3H1, and MTR4 (Figure 5E, black bars). 296 THOC2 functions in RNA export, ZFC3H1 functions as a linker between the PAXT and the 297 nuclear RNA degradation complex, and MTR4 is a helicase required for the function of all three 298 known nuclear RNA degradation complexes ${ }^{14}$. Other members of the PAXT complex were 299 identified including PABPN1 and ZC3H18. In cells expressing CDK13 ${ }^{\text {mel }}$, ZC3H14 lost 300 phosphorylation at S475 (as determined by mass spectrometry) (Figure S5A) and exhibited 301 decreased binding to THOC2 $(\mathrm{q}<0.0001)$, ZFC3H1 $(\mathrm{q}<0.0001)$, and MTR4 $(\mathrm{q}=0.018)$ but stable 
binding to PABPN1 and ZC3H18 (Figure 5E, blue bars). Together, these data show that CDK13 normally activates the recruitment of the PAXT complex to prematurely terminated polyadenylated transcripts via phosphorylation of the ZC3H14 adaptor. Upon loss of CDK13

305 function, the PAXT complex is not efficiently recruited to truncated mRNAs, resulting in their stabilization and translation into truncated polypeptides that promote melanomagenesis (Figure

\section{S5B).}

\section{Discussion}

Our data show that CDK13 functions as a tumor suppressor in melanoma and that CDK13 ${ }^{\text {mel }}$

310 alleles promote melanomagenesis through a dominant negative mechanism requiring CCNT1.

311 We provide evidence that $C D K 13$ mutation or loss leads to a failure to degrade prematurely

312 terminated transcripts. We found that CDK13 binds to, and may phosphorylate ZC3H14, a

313 component of the PAXT complex which is responsible for degrading polyadenylated

314 prematurely terminated RNAs. Consistent with the functional link to CDK13 that we have

315 established, ZC3H14 germline mutations also cause a neurodevelopmental disorder ${ }^{37}$. We

316 confirmed that ZC3H14 associates with the PAXT nuclear RNA exosome complex as recently

317 reported $^{14}$. Upon CDK13 ${ }^{\text {mel }}$ expression, we showed that ZC3H14 lost phosphorylation at S475,

318 which coincided with reduced recruitment of ZFC3H1 and MTR4. Because PAXT has been

319 shown to specifically target polyadenylated prematurely terminated RNAs for nuclear

320 degradation ${ }^{15}$, we suppose that CDK13 normally phosphorylates ZC3H14 to recruit the PAXT

321 complex to degrade aberrant RNAs made during failed transcriptional elongation. When $C D K 13$

322 is mutated, failure to recruit the PAXT complex results in the accumulation of truncated

323 transcripts which are in turn translated into truncated proteins, contributing to aggressive

324 melanoma (Figure S5B). 
The proteomic data suggest prematurely terminated transcripts that accumulate in CDK13 ${ }^{\text {mel }}$

326 expressing cells are translated into truncated proteins. We hypothesize that the acceleration of

327 melanoma progression we observe in tumors expressing CDK13 ${ }^{\text {mel }}$ could be influenced by the

328 presence of these aberrant truncated proteins. Some of the proteins showing evidence of

329 truncation in our data are tumor suppressors that would be expected to lose functionality; others

330 may act in a dominant negative fashion to favor tumor progression ${ }^{29-31}$.

331 Recent reports suggest that prematurely terminated RNAs may be a newly recognized and

332 more generalized oncogenic mechanism. Widespread intronic polyadenylation events were

333 reported to disrupt tumor suppressor genes in leukemia by generating truncated mRNAs and

334 proteins, however the truncation mechanism was not defined ${ }^{38}$. Loss of function mutations in

335 CDK12 in metastatic castration-resistant prostate cancer and serous ovarian carcinomas were

336 reported to cause increased production of truncated RNAs in DNA repair genes ${ }^{28,39}$. The tumor

337 suppressor function of CDK13 appears to uniquely function to target truncated messages for

338 degradation via PAXT-dependent surveillance, representing an additional mechanism by which

339 the oncogenic activity of prematurely terminated RNAs is countered in normal cells.

340 Our data are consistent with the evolutionarily related CDK12 and CDK13 having related

341 but distinct functions (Table S1). Indeed, as known tumor suppressors their roles could be

342 complementary, with CDK12 preventing the early termination of transcripts and CDK13

343 promoting the degradation of any prematurely terminated message that escape CDK12-mediated

344 suppression.

345 CDK13 was recently identified as being required for proper development of neural crest-

346 derived tissues. New truncating mutations in $C D K 13$ cause syndromic congenital heart disease ${ }^{11}$

347 and new kinase domain mutations identical to those we identified in melanoma cause a 
developmental disorder that affect heart, brain, and craniofacial development ${ }^{10}$. As melanoma is a cancer of neural crest derived melanocytes, neural crest derived tissues may have a particular sensitivity to CDK13 mutation, perhaps due to their broad differentiation potential and exquisite sensitivity to transcriptional changes. Our findings have prognostic implications for patients with CDK13-altered melanoma and suggest that patients with mutated or downregulated CDK13 should be considered for early stage adjuvant therapy trials. Our data suggest that patients with a CDK13-related developmental disorder should be monitored for melanoma.

\section{Methods:}

357 Data Availability: The datasets generated during and/or analyzed during the current study are 358 uploaded to GEO. The TCGA patient RNA-sequencing bams controlled-access data so they cannot be publically shared as they contain unique identifiers. The TCGA patient IDs used for analysis are listed in Supplementary Table 4.

Code Availability: Custom code is available upon request.

362 Patient mutation and survival analysis: Overall survival, mutation, and expression data (z-

363 score values) from the TCGA melanoma cohort 40 were downloaded on 4/7/17 using cBioPortal $^{17}$. A low-function CDK13 group was defined by either having a mutation in CDK13 or having low CDK13 expression, which was defined by z score equal to or less than -1.0. The difference in survival between low-function CDK13 vs. remaining cases was tested using a logrank test. The difference in survival between low-function CDK13 stage 0, 1, or 2 patients were compared with remaining stage 1 or 2 patients. Mutation plot was downloaded on 4/7/17 and percent mutations were calculated with all data sets available 4/7/17 using cBioPortal $^{17}$, excluding datasets from the Broad Institute, as it has duplicate samples with the DFCI/Broad set.

371 Zebrafish model: All Experiments were performed in accordance with relevant guidelines and

372 regulations. Animal studies were approved by Boston Children's Hospital Institutional Animal

373 Care and Use Committee (Protocol 17-10-3530R). Experiments were performed as

374 published ${ }^{19,21}$. Briefly, p53/-; mitfa:BRAFV600E;Na-/- one-cell embryos were injected with

375 either 20ng/uL control or experimental MiniCoopR (MCR) DNA along with tol2 in vitro 
transcribed RNA for integration. In overexpression experiments, control vectors expressed EGFP. In CRISPR experiments, control vectors expressed a gRNA to arhgapl la, a gene whose knock out has the same tumor curve as no gRNA vector in our system. In experiments where more than one vector was injected, DNA was prepared at 20ng/uL but divided equally between included vectors. For most experiments, embryos were sorted for melanocyte rescue at 5dpf. For $c d k 13$ CRISPR, control CRISPR, CDK13 WT expression, and $c d k 13$ CRISPR + CDK13WT expression embryos were sorted for melanocyte rescue, the 20 embryos with the most rescue were collected, and they were imaged at $3 \mathrm{dpf}$. In all experiments, 20 zebrafish were raised per tank to control for density effects. Zebrafish were scored for the emergence of raised melanoma lesions as published ${ }^{21}$. Melanoma-free survival curves and Log-rank tests were generated in Prism. As melanocyte-specific $c d k 13$ CRISPR/Cas9 zebrafish and melanocyte-specific CDK13 WT expressing zebrafish had few melanocytes as adults, tumor curves were not gathered. All zebrafish regardless of sex were included as zebrafish sex is environmentally determined and not apparent until after most of the time points observed.

390 Mutation Visualization on Crystal Structure: Patient mutations in CDK13 were plotted on the 391 crystalized kinase domain ${ }^{18}$ using PyMOL.

\section{In vitro Kinase Assays:}

393 Radioactive kinase reactions were performed using recombinant human CDK13 (694-1039) and

394 Cyclin K (1-300) proteins. Purified recombinant wild type CDK13/CycK and mutant

395 CDK13(R860Q)/CycK, CDK13(W878L)/CycK, and CDK13(K734R)/ CycK at a concentration

396 of $0.2 \mu \mathrm{M}$ were assayed for phosphorylation activity in a final volume of $30 \mu \mathrm{L}$ containing

397 kinase buffer (40 mM HEPES, pH 7.5, 34 mM NaCl, 34 mM KCl, 10 mM MgCl2, 5\% glycerol), 398 CDK13 substrates (GST-CTD f.1. $10 \mu \mathrm{M}$ ), cold ATP (to a final concentration of $1 \mathrm{mM}$ ) and 3 $399 \mathrm{mCi}\left[{ }^{32} \mathrm{P}\right]-\gamma$-ATP. The reaction mixture was incubated for $30 \mathrm{~min}$ at $30^{\circ} \mathrm{C}$ at 350 r.p.m. and 400 terminated by adding EDTA to a final concentration of $50 \mathrm{mM}$. Aliquots of $15 \mu \mathrm{l}$ each were 401 spotted onto P81 Whatman paper squares. Paper squares were washed three times for 5 min with $4020.75 \%(\mathrm{v} / \mathrm{v})$ phosphoric acid, with at least $5 \mathrm{ml}$ washing solution per paper square. Radioactivity 403 was counted in a Beckman Scintillation Counter (Beckman-Coulter) for max. 5 min. Kinase 404 assays were performed in duplicate and are represented as mean with s.d. One-way ANOVA for 405 c-Myc substrate had an "F" value of 1084 and 4 degrees of freedom (dof). One-way ANOVA for 406 CTD52 had an "F" value of 195 and 4 dof. 
Cloning (all except for mouse ES cells):

408 MiniCoopR (MCR) system ${ }^{19,21}$ : Zebrafish MCR overexpression constructs using the mitfa

409 promoter, relevant coding sequence, 3' pA tail, and the $M C R$ destination vector were assembled

410 with LR Clonase II Plus (Thermofisher, 12538120).

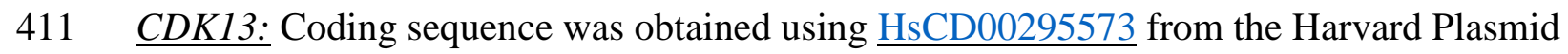

412 Repository (https://plasmid.med.harvard.edu/) and sequence verified. CDK13 in vitro

413 mutagenesis for K734R, R860Q, P869S, W878L, P881L, and P893L were performed using

414 primer design from www.genomics.agilent.com/primerDesignProgram.jsp, Q5 hot start

415 polymerase with GC enhancer (NEB M0493S), and DpnI digest.

416 ZC3H14: Full-length nuclear isoform ZC3H14 coding sequence was obtained from Dharmacon

417 (Accession: BC011793 Clone ID: 4298961). In vitro mutagenesis of S475D was done using the

418 NEB site directed mutagenesis kit (NEB E0554S).

419 CRISPR/Cas9 Modified MCR system: The modified CRISPR MCR vector used as reported ${ }^{19}$.

420 Briefly, CRISPR/Cas9 MCR constructs include a mitfa promoter driving Cas9 and a U6

421 promoter driving expression of the gRNA. gRNAs to $c d k 13$, arhgapl la (control), ccnT1, or

$422 \operatorname{ccnK}$ were selected using $\mathrm{CHOP} \mathrm{CHOP}^{41,42}$ in the beginning of exons predicted to code for

423 required protein domains. Prior to cloning into the in vivo system, multiple gRNAs were tested

424 for cutting efficacy. To test cutting efficiency, in vitro synthesized gRNA [oligos were annealed

425 and extended with T4 DNA polymerase and then synthesized with T7 RNA polymerase using

426 the MEGAscript T7 kit (Thermofisher, AM1333)] and Cas9 protein (PNA Bio, CP01) were

427 injected into one-cell stage zebrafish embryos. DNA was extracted at 48 hours using the

428 HotSHOT method ${ }^{43}$, PCR across the gRNA site was done, and a T7E1 assay was used to assess

429 cutting ${ }^{41}$. gRNAs with the most cutting by T7E1 were cloned into the MCR CRISPR vector

430 using a BseRI and injected into the one cell stage. Subsequent workflows are described in other

431 sections. CRISPR tumor analysis was done by extracting DNA from melanomas using the

432 HotSHOT method ${ }^{43}$. PCR with a proofreading polymerase (NEB M0503S) was used to amplify

433 across the cut site, and samples were submitted to the MGH DNA Core for CRISPR sequencing.

434 Data sets were mapped to the Danio rerio genome (version GRCz11) using Bowtie (version

435 0.12.9). CrispR Variants Lite was used to identify insertions and deletions (indels) around the

436 gRNA site (http://imlspenticton.uzh.ch:3838/CrispRVariantsLite/). Indels at the gRNA cut site in

$437>1 \%$ of reads were used in downstream calculations. Reads that were predicted to maintain 
438 function included in-frame indels as well as wild type reads. Reads that were predicted to cause 439 loss of function included out-of-frame indels.

440 Zebrafish brightfield imaging: Zebrafish at 3 day, 2 week, 4 week, and 9 week timepoints were 441 assessed for pigmentation and tumor onset via brightfield microscopy (Nikon DS-Ri2).

442 Maximum backlight and LED illumination (NII-LED) settings were utilized with 0.5X

443 magnification lens [1X/2X/3X zoom for 9/4/2 weeks post fertilization (wpf) zebrafish]. 3 day

444 post fertilization (dpf) zebrafish were transferred to a $35 \mathrm{~mm}$ dish, swirled, and photographed

445 with about 20 fish in a photo at once with $0.5 \mathrm{X}$ magnification lens and $3 \mathrm{X}$ zoom. Melanocytes

446 were quantified from $3 \mathrm{dpf}$ zebrafish photos and normalized to the number of zebrafish counted.

447 One-way ANOVA was done. "F" = 13.14 and degrees of freedom = 3. Melanocyte patterns from

4489 wpf were categorized into a) no melanocytes, b) minimal melanocytes (0-33\% of zebrafish

449 length covered), c) strong melanocytes (34-100\% of zebrafish length covered), or d) black patch

450 (disrupts normal stripes and cross section is $\geq$ the distance between that zebrafishes eyes.). All

451 zebrafish were anesthetized in tricaine solution (3-amino benzoic acidethylester, 4g/L) and

452 oriented in an imaging mold (2\% agarose in E3 buffer) prior to image capture.

453 Human cell lines: A375 human melanoma cells were identity-verified

454 (http://moleculardiagnosticscore.dana-farber.org/human-cell-line-identity-verification.html) and

455 then used for transient transfections for IP-MS or knockdown of CDK13. Mycoplasma testing

456 was done within one week of every experiment using human cell lines (Lonza, Mycoalert PLUS,

457 LT07-710). All cell lines were always mycoplasma negative. Cells were grown in DMEM

458 supplemented with 10\% FBS, penicillin/streptomycin antibiotics, and L-glutamine.

459 Expression for IP-mass spectrometry: LR Clonase II Plus was used to assemble pcDNA3.2 C460 terminal V5 tag destination vector (ThermoFisher 12489019) with either CDK13 WT, CDK13

461 W878L, CLOVER, or ZC3H14 in Constructs were transiently transfected using Lipofectamine 4623000 per instructions (Thermofisher, L3000008).

463 Stable Human Cell Lines. LR Clonase II Plus was used to assemble CLOVER, CDK13 WT, $464 C D K 13$ R860Q, and CDK13 W878L into pLenti CMV Blast DEST. pLenti CMV Blast DEST 465 (706-1) $)^{44}$ was a gift from Eric Campeau \& Paul Kaufman (Addgene plasmid \# 17451 ; 466 http://n2t.net/addgene:17451; RRID:Addgene_17451). Lines were made in biologic triplicate. 467 Cell counts were done at 24, 48, 72, and 96 hours. Cell doubling times calculated in exponential 468 growth phase. 
Westerns in human melanoma cells: Protein was quantified using DC Protein Assay (Biorad, 5000116). For CDK13 westerns, $20 \mu \mathrm{g}$ was run on 3-8\% tris acetate gel. Dry transfer was done at 25V for 10min. Primary anti-CDK13 antibody (Sigma HPA 059241, 1:1000) and primary loading control antibody anti-VCL (Sigma HPA 002131, 1:2500) were incubated overnight. For CLOVER, anti-GFP (Santa Cruz sc-9996, 1:1000) was incubated overnight. Rabbit or mouse secondary antibodies were incubated for $1 \mathrm{hr}$ at RT (CST 7074S or 7076S, 1:2000). Films were developed with Pierce ECL substrate (thermofisher, 32106). ChIP-sequencing:

477 Zebrafish with melanomas expressing either EGFP or CDK13 W878L in mitfa:BRAFmut+/+; 478 p53-/-; Na-/- zebrafish were sacrificed on ice and the tumors were dissected. 11 EGFP or 6 479 CDK13 W878L expressing melanomas were isolated at the same time point. Tumors were 480 combined and minced. Cells were collected and fixed in 1.1\% formaldehyde solution for 10min 481 at room temperature (RT). Tumors were homogenized and filtered through a $100 \mu \mathrm{m}$ filter, spun 482 down, washed with $1 \mathrm{X}$ PBS, and cells were counted using a Neubauer Chamber (9.00E+07 for $483 C D K 13$ W878L and 7.10E+07 for EGFP expressing melanomas). Cells were spun again and the 484 pellet was frozen with a dry ice/ethanol bath. ChIP was performed as published ${ }^{45}$, except upon 485 lysis spike in Drosphila chromatin was added with 10ng per million cells (Active motif, 53083) 486 and spike-in antibody $2 \mu \mathrm{g}$ was added to the beads (Active Motif, 61686). Briefly, IPs were 487 performed using antibodies to CDK13 (Bethyl, A301-458A), hypophosphorylated RNAPII 488 (abcam ab817, clone 8WG16, lot GR313984-17), RNAPII S2 CTD (ab5095; Lot G309257-1). 489 Libraries were performed using the NEBNextMultiplex Oligos for Illumina kit (NEB) and run on 490 an Illumina HiSeq 2000.

491 For ChIP-seq from human melanoma cells, one replicate (line 1) of A375 human melanoma 492 cells expressing CLOVER and CDK13 R860Q as derived above in "Stable Human Cell Lines"

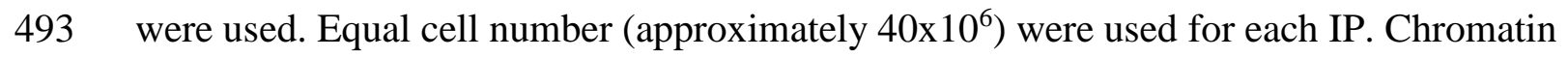
494 solubilization, spike in, and immunoprecipitation were performed using methods for zebrafish 495 melanomas as above.

\section{ChIP-Seq processing and quantification:}

497 Raw reads were aligned twice: first to the dm6 revision of the D. melanogaster genome to 498 remove exogenous spike-in reads using bowtie version 1.2.2 ${ }^{46}$ with parameters $-\mathrm{k} 1$-m 1 ; 499 unmapped reads were then aligned to the danRer10 revision of the zebrafish genome with default 
parameters or the non-random hg19 revision of the human genome with parameters $-\mathrm{k} 2-\mathrm{m} 2$ and -1 set to read length. Display files were created using MACS ${ }^{47}$ with parameters -W -S space $=50-$ nomodel - shiftsize to get read counts in $50 \mathrm{bp}$ bins. These files were normalized to millions of mapped reads per sample and converted to TDF format using igvtools ${ }^{48,49}$ toTDF.

Version 90 of the danRer10 Ensembl gene set was used for zebrafish ChIP-Seq analysis, and hg19 RefSeq gene positions were downloaded as part of the ROSE package (https://bitbucket.org/young_computation/rose/). Reads-per-million-normalized ChIP-Seq signal was quantified in promoters (transcription start sites +/- 250bp) using bamToGFF with parameters $-\mathrm{m} 1-\mathrm{r}-\mathrm{e}-200-\mathrm{f} 1$. Log 2 fold-changes in signal were performed after adding one pseudocount to each condition. For displays of single-bin coverage, corresponding input signal calculated identically was subtracted from each region, and only regions with a positive inputsubtracted CDK13 signal were shown.

512 Whole-gene metagenes across all genes greater than $2 \mathrm{~kb}$ in length were constructed in three 513 sections: $-2 \mathrm{~kb}$ upstream of the transcription start site to the transcription start site, transcription

514 start site to the transcription end site, and transcription end site to $2 \mathrm{~kb}$ downstream of the

515 transcription end site. Matrices of coverage were calculated using bamToGFF

516 (https://github.com/BradnerLab/pipeline) with parameters -m 50 -e 200 -r -f 1 for the upstream

517 and downstream regions, and -m 150 -e 200 -r - $f 1$ for the genic region. The values in each bin 518 were averaged across the genes used.

519 Gene lists were subsetted and/or broken into equally sized groups using different

520 characteristics for different analyses. Unless otherwise noted, only genes greater than $2 \mathrm{~kb}$ from 521 their transcription start sites to their transcription end sites were used.

522 Fold-change metagenes used the subset of all genes regardless of length that were in the $20 \%$ 523 whose fold-changes in promoter CDK13 levels increased the most dramatically. The displayed 524 metagenes represent per-bin $\log 2$-fold ratios of the average values for Ser2-RNA Polymerase II 525 vs. the average values for total RNA Polymerase II where one pseudocount was added.

526 To study the effects of CDK13 mutation on coverage of genes expressed at different levels, 527 the gene list was broken into six equally sized groups by their mean expression across three 528 control samples for zebrafish samples. For human samples, the gene list was broken into five 529 equally sized groups and expression amounts were determined from ribo-depleted RNA- 
sequencing from the three CLOVER expressing A375 lines as described above in "Stable

\section{1}

532

533

534

535

536

537

538

539

\section{Human Cell Lines".}

To study the effects of CDK13 mutation on coverage of genes of different lengths, genes were broken into six equally sized groups based on their gene length from transcription start site to transcription end site.

bigWig files displaying read coverage were created using readGAlignments and coverage from the GenomicAlignments ${ }^{50}$ package and export.bw from the rtracklayer ${ }^{51}$ package, where putative PCR duplicate reads were removed. IGV (http://www.broadinstitute.org/igv) was used to view bigWig files in figures.

For anti-CDK13 ChIP-sequencing from melanomas from mitfa:BRAFmut+/+; p53-/-; Na-/zebrafish expressing either CDK13 R860Q + ccnTl CRISPR or CDK13 R860Q + ctrl CRISPR, coverage of zebrafish promoters was quantified by bamToGFF. Gene positions for the GRCz10 revision of the zebrafish genome were downloaded from Ensembl (version 90). Two different windows were used: $+/-2 \mathrm{~kb}$ from the transcription start sites is displayed, and $+/-250 \mathrm{bp}$ from the transcription start sites was used to order rows/regions. Regions for display were broken into 100 equally sized bins (-m 100 -r ), whereas ranking was done using a single window (-m 1 -r). In each case, input signal was subtracted from the corresponding regions. Promoters were subsetted for those that had background-subtracted RPM values in the $500 \mathrm{bp}$ window $>1$. They were ordered by their $\log 2$ fold-change between $\mathrm{sgCtrl}$ and sgccnT1 background-subtracted coverage, with one pseudocount added.

\section{Zebrafish Melanoma RNA-seq and RNA-seq processing:}

Zebrafish melanomas expressing EGFP, K734R, or R860Q in mitfa:BRAFmut+/+; p53-/-; Na/- zebrafish were sacrificed on ice and the tumors were dissected, homogenized in RLT buffer, subjected to QIAshredder columns (Qiagen, 79656), and then RNA was isolated with a column based method with genomic DNA column removal (Qiagen, 74134). RNA was ribodepleted (Illumina Ribozero Gold, MRZG12324). Ribodepletion was confirmed with Agilent 4200 Tapestation. Library prepped with random priming (NEBNext Ultra RNA Library Prep Kit for Illumina, E7530), fragmentation time of 15min, 12 PCR cycles, and sequenced on an Illumina HiSeq 2000 (100bp paired end reads). Sequenced reads were mapped to a custom version of the danRer10 transcriptome where all chromosomes were included plus human CDK13 using tophat 2.1.152 with parameters -library- 
561 type fr-unstranded -no-novel-juncs and -G set to a GTF of Ensembl zebrafish genes (v90).

562 Expression quantification was performed using htseq-count ${ }^{53}$ using the same GTF as above and

563 parameters -r name -i gene_name -stranded=no -f bam -m intersection-strict. Gene-level

564 transcription read counts were then normalized to transcripts per million (TPM) (counts / bp in

565 exons of all isoforms / 1000 / total counts across all genes / 1000000). Differential expression

566 analysis was performed using DEseq $2^{54}$ with standard parameters comparing raw read counts in

567 all overexpression mutant samples against all overexpressed EGFP samples.

568 Mouse Embryonic Stem Cells:

569 Cell Culture, Cell Line Generation, Drug Conditions:

570 All cell lines were tested for mycoplasma contamination periodically, including immediately 571 after generation of CRISPR-modified clonal cell lines via the MycoAlert Mycoplasma Testing

572 Kit (Lonza). Results were always negative for mycoplasma contamination. V6.5 (C57B1/6-129)

573 mESCs and derived cell lines were cultured on $0.2 \%$ gelatin-coated tissue culture plates in ES

574 media: Dulbecco's Modified Essential Media (Thermo Fisher) buffered with 10mM HEPES

575 (Thermo Fisher) and supplemented with 15\% Fetal Bovine Serum (Hyclone), 1000U/mL

576 leukemia inhibitory factor (Millipore), 1x non-essential amino acids (Thermo Fisher), 2mM L-

577 glutamine (Thermo Fisher), 0.11mM ß-mercaptoethanol (Sigma), $100 \mathrm{IU}$ penicillin and

$578 \quad 100 \mathrm{ug} / \mathrm{mL}$ streptomycin (Corning).

579 Cdk13 $\Delta$ clones were generated using CRISPR/Cas9 genome editing technology as follows

580 (see Figure S3B). sgRNAs targeting intron 3 and intron 4 of the endogenous Cdk13 locus were 581 cloned into pX458 (a gift from Feng Zhang, Addgene plasmid \#48138) ${ }^{55}$ or pX330 (a gift from

582 Feng Zhang, Addgene\# 42230) ${ }^{56}$ respectively (see Supplementary Table. 3 for sequences). Two

583 independent pairs of sgRNAs (one sgRNA targeting intron 3 and one sgRNA targeting intron 4)

584 were co-transfected into wildtype V6.5 mouse embryonic stem cells with Lipofectamine®2000

585 (Thermo Fisher), and single-cell sorted for GFP+ fluorescence (transfected cells) 24 hours after

586 transfection. Clones were screened for homozygous deletion of exon 4 by PCR and confirmed by

587 sanger sequencing. Knockout of endogenous Cdk13 was confirmed by Western blot (Figures

588 S3C). One knockout clone from each sgRNA pair was used throughout the study to control for 589 off-target effects of the sgRNAs.

590 A Dox-inducible Cdk13 transgene was stably introduced into the two Cdk13 $\Delta$ clones used 591 throughout this studying using a piggybac retrotransposon system. N-terminal Flag- HA- tandem 
epitope-tagged Cdk13 (NP_001074527.1 isoform) was cloned via overlap extension PCR using

593 two templates: (1) a synthetic gene block containing the codon-optimized N-terminus of Cdk13

594 (first 1577 base pairs) to reduce the high GC content in the region and (2) polyA-selected mouse

595 cDNA from V6.5 cells. This PCR product was cloned into pCR8/GW/TOPO (Thermo Fisher)

596 followed by transfer into the doxycycline-inducible piggybac expression vector, PBNeoTetO-

597 Dest (a gift from A.W. Cheng), using standard TOPO and Gateway cloning kits (Thermo Fisher).

598 The final Cdk13 transgene sequence is provided in Supplementary Table 5. This expression

599 vector was cotransfected with pAC4 (constitutively expressing M2rtTA, the Dox-inducible

600 transactivator, flanked by piggybac recombination sites, A.W. Cheng) and mPBase (piggybac

601 transposase expression plasmid, A.W. Cheng) using Lipofectamine®2000. 24 hours after

602 transfection, cells were selected with $150 \mathrm{ug} / \mathrm{mL}$ Hygromycin (Thermo Fisher) and $200 \mathrm{ug} / \mathrm{mL}$

603 G418 (Sigma) to select stable transformants and subsequently, single-cell cloned. Clones were

604 screened for near wild-type levels of Cdk13 expression upon addition of 1ug/mL Dox for 24

605 hours. Two clones (one from each sgRNA pair used for knockout) were isolated that expressed

606 near wild-type levels of Cdk13 expression upon addition of 1ug/mL Dox for 24 hours

607 (representative Western blot for one clone shown in Figure S3E), and these two clones were used

608 throughout the study.

609 Western Blotting

610 Whole cell extract was harvested by washing the cells in cold phosphate-buffered saline

611 (PBS) and lysing in RIPA (10mM Tris pH7.4, 150mM NaCl, 1\% TritonX-100, 0.1\% SDS, 0.5\%

612 Sodium Deoxycholate, and 1mM EDTA) supplemented with 1x cOmplete, EDTA-free Protease

613 Inhibitors (Roche) and 2uL/mL Benzonase Nuclease (Sigma). Lysates were incubated on ice for

614 at least $30 \mathrm{~min}$, centrifuged for $10 \mathrm{~min}$ at $4{ }^{\circ} \mathrm{C}$ at max speed, and the cleared lysate was quantified

615 using a standard BCA assay (Thermo Fisher). Lysates were normalized for equivalent total

616 protein in 1x Loading Dye (62.5mM Tris pH6.8, 5\% glycerol, 2\% SDS, 16.67\% BME, and

$6170.083 \%$ bromophenol blue) or 1x NuPAGE LDS Sample Buffer (Thermo Fisher) with 1x

618 NuPAGE Reducing Agent (Thermo Fisher). Normalized lysates were boiled for 5 min and run

619 on NuPAGE 4-12\% Bis-Tris Gels (Thermo Fisher). Gels were transferred overnight (30 V) to

620 PVDF in 10\% methanol supplemented 1x NuPAGE Transfer Buffer (NuPAGE Bis-Tris Gels).

621 Primary antibodies used for blotting: Anti-HA High Affinity Antibody (Roche 11867423001),

622 CDK13 (a gift from Arno L. Greenleaf), Enolase I (CST 3810S), alpha-Tubulin (Genescript 
623 a01410). Secondary antibodies used: ECL Anti-Rat IgG (GE Healthcare NA935V), ECL Anti-

624 Mouse IgG (GE Healthcare NA931V), and ECL Anti-Rabbit IgG (GE Healthcare NA934V).

625 RNA Sequencing:

626 Two independent Cdk13 $\Delta$ clones with Dox-inducible Cdk13 were pre-treated with $1 \mathrm{ug} / \mathrm{mL}$

627 Doxycycline (Dox) daily for at least 5 days prior to the start of the time course to express

628 complementing levels of Cdk13 transgene in the Cdk13 $\Delta$ background (Figure S3D). Dox was

629 withdrawn from these cells at time 0, which resulted in significant knockdown and undetectable

630 levels of Cdk13 after 48- or 72- hours respectively (Figure S3E). RNA was harvested in

631 biological duplicate from both independent clones from cells maintained in Dox at time 0

$632(+\mathrm{Cdk} 13)$ or withdrawn from Dox for 48 or 72 hours using Trizol (Thermo). RNA was extracted

633 following the standard Trizol protocol and subsequently DNase treated with Turbo DNase

634 (Thermo Fisher) under standard reaction conditions. RNA quality was assessed by Agilent 2100

635 Bioanalyzer and only samples with a RIN value $\geq 8.9$ were used for library preparation and

636 sequencing. PolyA-selected libraries were made from 1ug of total RNA input using the TruSeq

637 Stranded mRNA Library Prep Kit (Illumina RS-122-2102) with multiplexing barcodes,

638 following the standard protocol with the following specifications: (1) 5 min RNA fragmentation

639 time, (2) Superscript III (Thermo Fisher) was used for reverse transcription, (3) 15 cycles of PCR

640 were used during the library amplification step, and (4) AMPure beads (Beckman Coulter) were

641 used to size select/purify the library post PCR amplification instead of gel size selection. The 12

642 libraries were pooled and sequenced (75 base pair, paired-end reads) on one flow cell of an

643 Illumina NextSeq500.

644 5' to 3' RNA-seq gradient analysis:

645 For patient data, were approved via dbGaP for access to RNA-seq bams. RNA-seq bams were 646 downloaded on 7/27/18 from cutaneous melanoma patients from The Cancer Genome Atlas

647 (TCGA) ${ }^{40}$ from 3 patients with $C D K 13$ kinase-domain mutations and 5 best matched control WT

648 CDK13 patients by 1) stage, 2) age, 3) sex, and 4) oncogene from the National Cancer Institute

649 (NCI) Genomic Data Commons (GDC) Data Portal (Patient characteristics in Supplementary

650 Table 4). For zebrafish melanomas, bams from above for $C D K 13 R 860 Q(\mathrm{n}=5)$ and EGFP $(\mathrm{n}=4)$

651 melanomas were used. For mouse ES cells, bams from above RNA-seq were used.

652 For each genome analyzed, The files were sorted using SAMtools ${ }^{57}$. All exons were assigned 653 to one of the following classes: F: first exon, 5'-most exon in any transcript of the gene; AF: the 
654 first exon in an annotated transcript that is not the 5'-most exon in that gene; I: Internal exon,

655 AL: the last exon in an annotated transcript that is not the 3'-most exon in that gene; L: last exon,

6563 '-most exon in all transcripts of the gene. The following annotations were used: human,

657 Gencode version 28 gtf; zebrafish, GRCz10; mouse, custom annotation generated with custom

658 scripts. For each transcript in the input annotation, the exons were sorted by position and the first

659 exon in each transcript was assigned the value 'AF' and the last AL for "Alternative First/Last".

660 All others are I for "Internal". After all transcripts belonging to a gene were processed, the

661 complete list of exons was sorted by position to determine the 5'-most and 3'-most exons, which

662 were assigned " $F$ " and "L" status; all other exons retained their originally assigned

663 classifications. All exons were then subjected to differential expression analysis using DEXSeq

66458,59 . The log2-fold changes in exons belonging to each class were plotted and the differences in

665 the distribution of these differential expression comparisons were determined using the non-

666 parametric Wilcoxon rank sum test, two-sided.

667 IGV (http://www.broadinstitute.org/igv) was used to generate TDF files from human patient

668 data and was used to view RNA in Figure 3E, signal normalized to $4^{\text {th }}$ exon as difference

669 between internal exons did not change.

\section{Distal pA Transcripts per Million Analysis:}

671 Fastq files of reads from polyA-selected, strand-specific RNA sequencing that corresponded to

672 the 3' end of RNA fragments were first parsed to identify reads terminating in at least three

673 adenosines. The putative polyA tails were then trimmed from the reads, and the remaining

674 portion of the read was mapped, along with its mate pair, to the genome. Using custom scripts,

675 properly mapped reads were then filtered by assessing whether all of the trimmed 3' adenosines

676 were genomically encoded, in which case they were removed from further analysis, or

677 alternatively, represented evidence of posttranscriptional, non-templated polyadenylation in

678 which case they were kept. Kept reads were then intersected using bedtools with a set of

679 previously defined polyadenylation site clusters based on 3' end sequencing from mouse

680 embryonic stem cells of the same strain under steady-state conditions. Reads that overlapped

681 known polyadenylation sites were then intersected with gencode annotated 3' UTRs to identify

682 polyA reads corresponding to the distal polyA site (full-length mRNA). Using Rsem/EBseq, the

683 number of transcripts pertaining to each gene, normalized per million total transcripts estimated

684 for each sample, was used as an estimate of relative transcript abundance. We calculated the ratio 
685 of read counts assigned as described above to the distal, full-length mRNA polyadenylation sites

686 to the estimated transcripts per million (TPM) for each sample. The ratio of polyA reads/

687 transcript/ million for the four replicates of each genotype at each timepoint combined were then

688 plotted as a box-and-whisker plot and the significance level of differences in population

689 distributions were calculated by the Wilcoxon rank sum test.

690 Tandem mass spectrometry:

691 Protein was isolated from three separate EGFP and CDK13W878L zebrafish melanomas at week

69225.9 weeks post fertilization in RIPA buffer with BME and they were submitted to the Thermo

693 Fisher Scientific Center for Multiplexed Proteomics (TCMP) at Harvard

694 (https://tcmp.hms.harvard.edu/) for tandem mass spectrometry with isobaric tags and

695 synchronous precursor selection based MS3 technology. Peptides are selected for sequencing in

696 MS1 scans. MS2 spectra are used for identifying peptides, and MS3 spectra are used for

697 quantification via TMT reporter ions. MS2 spectra were searched using the SEQUEST algorithm

698 against a Uniprot composite database derived from the Danio Rerio proteome containing its

699 reversed complement and known contaminants. Peptide spectral matches were filtered to a $1 \%$

700 false discovery rate (FDR) using the target-decoy strategy combined with linear discriminant

701 analysis. Proteins were quantified only from peptides with a summed SN threshold of $>=100$ and

702 MS2 isolation specificity of 0.7 . Using these parameters, 46,853 peptides with an FDR of $<1 \%$

703 were identified which mapped to 6190 proteins.

704 Tandem mass spectrometry data analysis:

705 Data were filtered for peptides with unique identification. Peptide location was determined by

706 dividing the peptide start location by the protein length and $\log 2$ fold change between

707 CDK13W878L and EGFP was calculated. A D'Agostino and Pearson normality test $(p=0.3374)$

708 showed data were normal. To plot proteome-wide changes, individual peptides that had a two-

709 sided t-test $\mathrm{p}<0.1$ were binned and plotted by \% protein length (included 3676 measurements).

710 Linear regression showed a slope of -0.5349 and the $\mathrm{p}=0.0001$ that the slope was non zero.

711 To identify individual candidate proteins two methods were used. First, of the 3676 peptides

712 identified above, proteins that had >1 measurement and a $\log 2$ fold changed slope of $>-1$ were

713 included as candidates which identified 263 candidates. This method identified Separately, using

714 all unique peptides, any protein with $>3$ peptide measurements were plotted for slope. Proteins 
715 with a negative slope were tested with an F test $(\mathrm{p}<0.05)$ to determine good fit. This method 716 identified 103 candidates.

717 IP-mass spectrometry: pcDNA3.2 plasmids expressing CLOVER, CDK13 WT, CDK13

718 W878L, or ZC3H14 were transfected using lipofectamine 3000 (Thermofisher L3000008) per

719 protocol in $15 \mathrm{~cm} 2$ plate in duplicate or triplicate into either $\mathrm{A} 375 \mathrm{~s}$ or $C D K 13 \mathrm{R} 860 Q$ expressing

720 A375s.. 48 hours after transfection, nuclei were isolated (Thermofisher 78833) and lysed per

721 protocol with protease inhibitors with final volume of $500 \mathrm{uL}$. $500 \mathrm{uL}$ of non-denaturing lysis

722 buffer (NDLB =20mM Tris $\mathrm{pH} 8,140 \mathrm{mM} \mathrm{NaCl}, 10 \%$ glycerol, 1\%triton, 5mM EDTA) with

723 protease inhibitors (Sigma P8340) and phosphatase inhibitors [Na pyrophosphate (4mg/10mL)

724 and $\mathrm{Na}$ fluoride $(8 \mathrm{mg} / 10 \mathrm{~mL})]$ was added. V5-conjugated agarose beads (Sigma A7345) were

725 washed with phosphate buffered saline (PBS) per protocol and incubated overnight at 4C with

726 lysate. Beads were washed twice with NDLB with protease inhibitors and phosphatase inhibitors

727 and then four times with wash buffer with protease and phosphatase inhibitors $(150 \mathrm{mM} \mathrm{NaCl}$,

728 50mM Tris $\mathrm{pH}$ 8). Protein was boiled in Laemmli Buffer, run on an $8 \%$ tris/glycine gel, stained

729 with colloidal blue staining kit (Thermofisher LC6025), each lane was cut into 4 pieces, and each

730 gel fragment was submitted for mass spectrometry using the Taplin Mass Spectrometry Facility

731 at Harvard University. Proteins from CDK13 experiment were included in analysis if they were

732 identified in all IP replicates. IP'ed proteins were excluded from analysis if they had $>1$ peptide

733 in either control IP. Peptides for identified proteins were normalized as a percent of identified

734 CDK13 peptides and only proteins that averaged at least 1\% of CDK13 were plotted. $q$ values

735 were calculated with unpaired t test with multiple test correction using Prism software. Proteins

736 from $\mathrm{ZC} 3 \mathrm{H} 14$ experiment were included for analysis if they had at least 4 peptides in all

737 replicates of one condition and $>3 \mathrm{x}$ enrichment over control IP. We report here the 3 most

738 significantly changed proteins and 2 unchanged proteins from the same complex. Statistics were

739 done using multiple t-tests assuming similar scatter using Prism software.

$740 \quad$ ZC3H14 phosphorylation sites were reported if they were identified in all three biologic

741 replicates with either 1) modification score $>10$ or 2) >2 peptides calling the same site.

742 Zebrafish melanoma IHC: Zebrafish with melanomas were

743 sacrificed on ice, fixed in 4\%PFA 1xPBS 4-5d, washed in 1xPBS 2 days, washed in 50\% ethanol

7441 day, then stored in 70\% ethanol. CDK13 W878L and CDK13 P893L melanomas were isolated

745 at 18 weeks post fertilization (wpf). EGFP melanomas were isolated at 18 and 19 weeks post 
746 fertilization because there were not enough tumors to isolate all of them at $18 \mathrm{wpf}$. The first 10

747 melanomas with a gRNA to arhgapl la (control gRNA) were collected in batches as they arose

748 (6 at 13.7wks and 4 at 20.3 weeks) or gRNA to $c d k 13$ (first three at 19weeks and the last three at

749 26.3weeks). Zebrafish were paraffin embedded, sectioned, and stained for PH3 Serine 10 (CST

750 9701) at 1:200 and then stained with secondary HRP-goat anti rabbit secondary (Dako K5007)

751 by ServiceBio (http://www.servicebio.com). The square millimeter with the most PH3 positive

752 cells was identified and then the $\mathrm{PH} 3$ positive cells were counted per square mm.

754 Acknowledgements. This research was supported by Damon Runyon Cancer Foundation

755 Fellowship Award, American Society of Clinical Oncology Young Investigator Award, Charles

756 S Memorial Sloan Kettering Starr Foundation Cancer Consortium, The Hope Funds for Cancer

757 Research Grillo-Marxuach Family Fellowship, The American Lebanese Syrian Associated

758 Charities (ALSAC), R01CA103846-14, and T32HL116324 from the National Heart, Lung, and

759 Blood Institute. M.G. is a member of the excellence cluster ImmunoSensation (EXC2151) and

760 funded by a grant from the Deutsche Forschungsgemeinschaft (GE 976/9-2). The CDK13

761 antibody used for mouse experiments was a gift from A.L. Greenleaf. The pAC4 and

762 PBNeoTetO-Dest vectors were gifts from A. Cheng. S.D. was supported by a David H. Koch

763 Fellowship.

765 Author contributions. M.L.I. planned and performed the experiments with assistance from

766 K.Y.C., T.F., and C.W. with the following exceptions. Mouse ES cell experiments were

767 planned/performed by S.J.D. and kinase assays were planned/performed by S.D. M.L.I., B.J.A.,

768 P.B., S.B., and C.G.L. analyzed the data. D.L. coded the survival analysis. M.G. provided the

769 plots of the mutations on the CDK13 crystal structure and insight into the effects the mutations

770 could have on CDK13 function. K.A and T.H. advised on RNA experiments. R.A., P.S., P.B., 
771 and L.I.Z. supervised the project. M.L.I, B.J.A., P.B., and L.I.Z. wrote the manuscript.

772

773 Competing Interests. R.A.Y. is a founder and shareholder of Syros Pharmaceuticals, CAMP4

774 Therapeutics, Omega Therapeutics, and Dewpoint Therapeutics. P.A.S. is a founder and

775 shareholder of Syros Pharmaceuticals. L.I.Z. is a founder and stockholder of Fate Therapeutics,

776 CAMP4 Therapeutics, and Scholar Rock. He is a consultant for Celularity. B.J.A. is a

777 shareholder in Syros Pharmaceuticals.

779 Materials and Correspondence. Correspondence and requests for materials should be

780 addressed to L.I.Z. (zon@enders.tch.harvard.edu).

Table 1: CDK13 has a related but distinct biologic role and mechanism from CDK12.

\begin{tabular}{|c|c|c|}
\hline & CDK13 & CDK12 \\
\hline Mutated in Cancer & Melanoma & $\begin{array}{l}\text { Prostate }{ }^{60}, \\
\text { Ovarian } 61\end{array}$ \\
\hline $\begin{array}{l}\text { Mutated in } \\
\text { Developmental } \\
\text { Disorders }\end{array}$ & $\begin{array}{l}\text { New kinase domain mutations cause complex } \\
\text { disorder involving several neural crest tissues }{ }^{10,62} \text {, } \\
\text { New truncation mutations cause syndromic } \\
\text { congenital heart disease }^{11}\end{array}$ & Not detected \\
\hline Target genes & TNFalpha signaling & $\begin{array}{l}\text { DNA repair }{ }^{63} \\
\text { 28,39 }\end{array}$ \\
\hline RNA Polymerase II & $\begin{array}{l}\text { Not required for RNAPII CTD S2 } \\
\text { phosphorylation in melanoma }\end{array}$ & $\begin{array}{l}\text { Required for } \\
\text { RNAPII CTD } \\
\text { S2 } \\
\text { phosphorylation } \\
\text { in vivo } 28\end{array}$ \\
\hline RNA Mechanism & $\begin{array}{l}\text { Required for PAXT activation on prematurely } \\
\text { terminated RNAs }\end{array}$ & $\begin{array}{l}\text { Required to } \\
\text { suppress } \\
\text { intronic } \\
\text { polyadenylation } \\
28,39\end{array}$ \\
\hline
\end{tabular}




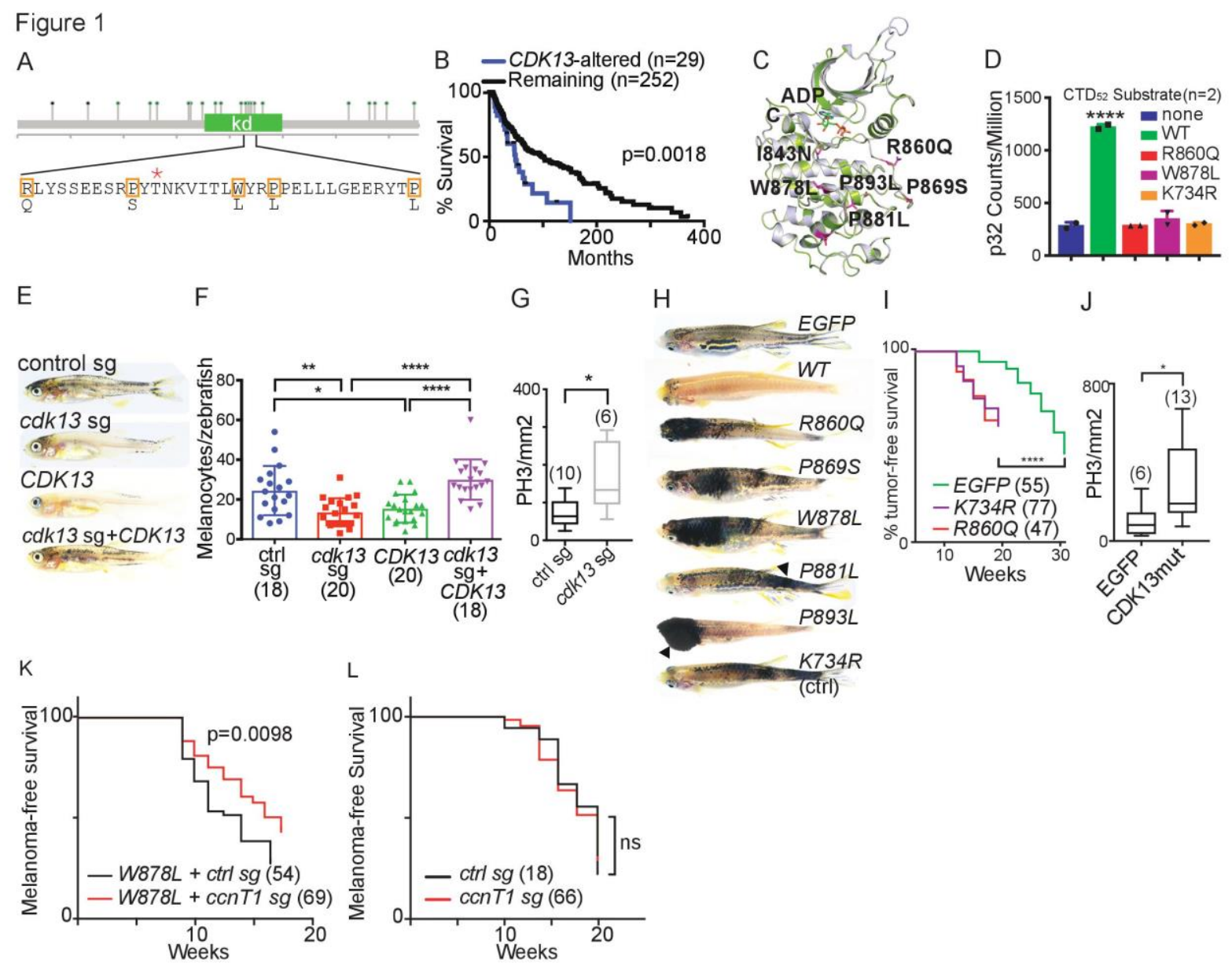

781 Figure 1: CDK13 is a melanoma tumor suppressor.

782 A) $C D K 13$ mutation plot in melanoma. Orange rectangles denote 5/7 mutations near the ATP-

783 binding site. $*$ =phosphorylation site. B) Survival plot of $C D K 13$ downregulation or mutation in

784 patients. $\mathrm{p}=0.0018$ Log-rank. $\mathrm{n}=$ patients. C) Patient kinase-domain mutations mapped on the

785 CDK13 crystal structure. Red residues = mutations. D) In vitro kinase assay of wild type and

786 patient-mutated CDK13 using full-length CTD $_{52}$ as the substrate. One-way ANOVA with no

787 kinase vs. all conditions; WT CDK13 $* * * *=\mathrm{q}=0.0001$, all mutated CDK13 comparisons non-

788 significant. Mean + SD. $\mathrm{n}=2$ replicates. E) Representative 4 week photos of mitfa:BRAF; p53-/-;

789 mitfa-/- zebrafish injected with 1) control gRNA, 2) $c d k 13$ gRNA, 3) overexpression of human

790 WT CDK13, or $c d k 13$ gRNA and overexpression of wild-type $h C D K 13$. F) Quantification of

791 melanocytes at 3 days post fertilization for mitfa:BRAF; p53-/-; mitfa-/- zebrafish injected with

792 1) control CRISPR (blue), 2) $c d k 13$ gRNA (red), 3) overexpression of human $C D K 13^{W T}$ (green),

793 or $c d k 13$ gRNA and overexpression of $C D K 13^{W T}$ (purple). $\mathrm{p}=$ one-way ANOVA, multiple 
794 comparisons. Mean +/-SD. $*=\mathrm{q}=0.186, * *=\mathrm{q}=0.0030, * * * *=\mathrm{q}<0.0001 .(\#)=$ zebrafish. G)

795 Phospho Histone H3 Serine 10 (PH3) antibody staining $/ \mathrm{mm}^{2}$ of melanomas from mitfa:BRAF;

796 p53-/-; mitfa-/- zebrafish with $c d k 13$ gRNA compared with control gRNA. p=0.014 (Mann

797 Whitney two-tailed t test). (\#)=melanomas. Box and whiskers plot, min to max. H) 9-week

798 photos from melanocyte-specific expression of 1) EGFP, 2) wild-type human $C D K 13,3-7)$

799 CDK13 patient mutations, and 8) CDK13 K734R (catalytically dead) in BRAF; p53-/-; mitfa-/-

800 zebrafish. Arrows=melanomas. I) \% melanoma-free survival of BRAF; p53-/-; mitfa-/- zebrafish

801 injected with melanocyte-specific expression of EGFP,CDK13 ${ }^{R 860 Q}$ (patient mutation), and

$802 C D K 13^{K 734 R}$ (catalytically dead). $* * * *=\mathrm{p}<0.0001$ (log-rank). (\#)=zebrafish. J) PH3 antibody

803 staining/mm² of melanoma from mitfa:BRAF; p53-/-; mitfa-/- zebrafish with EGFP vs. CDK13

804 mutant (W878L or P893L). p=0.0125 (Mann Whitney two-tailed t test). $(\#)=$ melanomas. Box and

805 whiskers plot, min to max. K) Melanoma-free survival of melanocyte-specific expression of

806 CDK13 W878L coinjected with melanocyte-specific CRISPR of either a control gRNA or ccnT1

807 gRNA. p=0.0098 (log-rank). (\#)=zebrafish. L) Melanoma-free survival of melanocyte-specific

808 expression of control gRNA or $c c n T 1$ gRNA alone. ns=non significant (log-rank). (\#)=zebrafish.

809 See also Figure S1. 
Figure 2

A
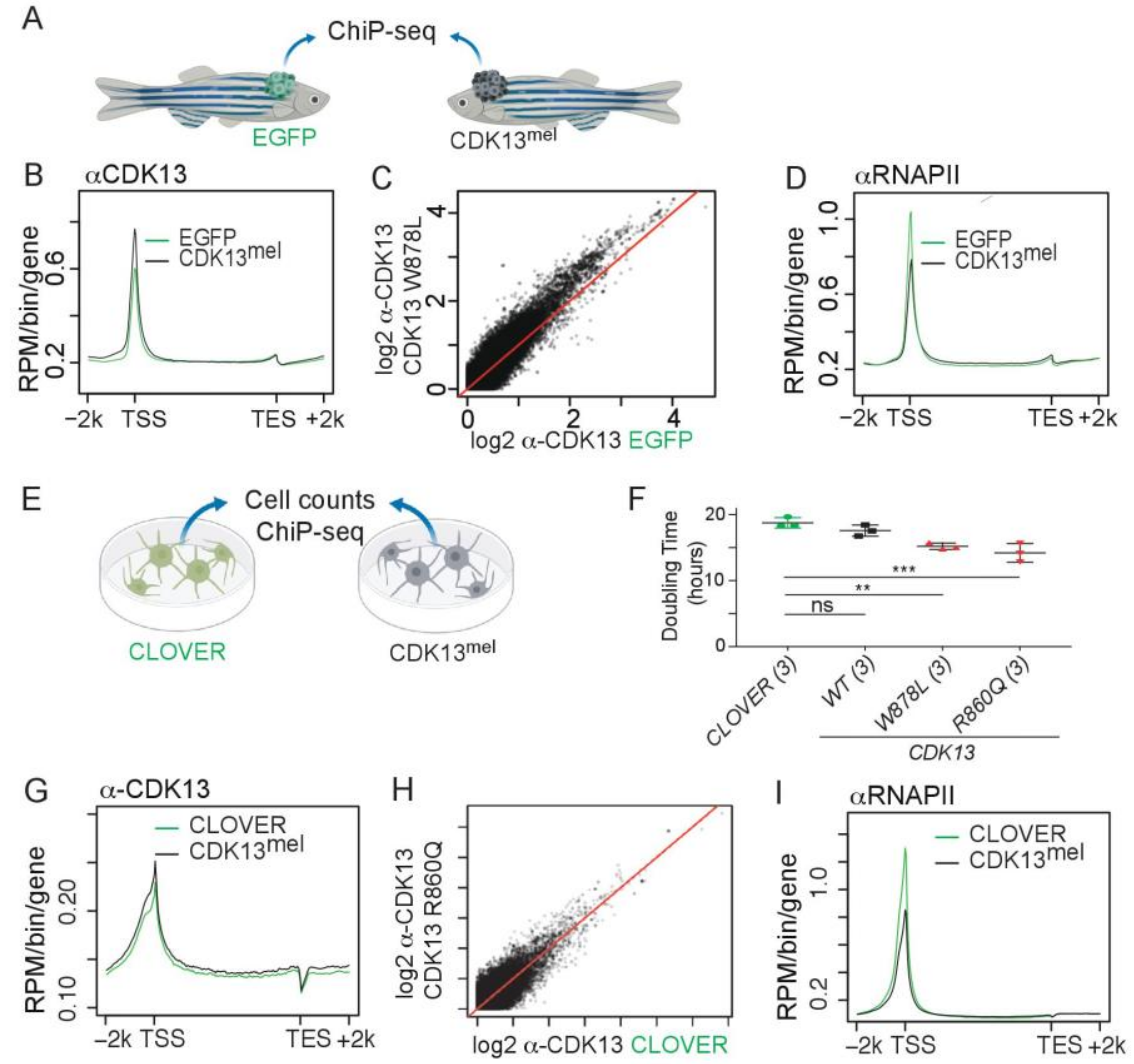

811 Figure 2: Mutant CDK13 binds chromatin in a dominant negative manner. A) Schematic

812 for zebrafish melanoma chromatin immunoprecipitation sequencing (ChIP-seq) of mitfa:BRAF;

813 p53-/-; mitfa-/- melanomas with melanocyte-specific expression of EGFP (green) or

$814 C D K 13^{W 878 L}$ (black). B-C) $\square$ anti-CDK13 zebrafish melanoma ChIP-seq displayed as a B)

815 metagene or C) $\log 2$ from EGFP vs. $C D K 13^{W 878 L}$ for each gene. D) anti-RNAPII zebrafish

816 melanoma ChIP-seq metagene. E) Schematic for ChIP-seq from human melanoma cells

817 expressing CLOVER fluorescent protein or CDK13 ${ }^{\mathrm{mel}}$. F) Doubling time for human melanoma

818 cell lines expressing CLOVER, CDK13 ${ }^{\mathrm{WT}}, \mathrm{CDK} 13^{\mathrm{W} 878 \mathrm{~L}}$, or CDK13 ${ }^{\mathrm{R} 860 \mathrm{Q}}$. One-way ANOVA

819 with CLOVER vs. all conditions. CDK13 ${ }^{\mathrm{W} 878 \mathrm{~L}} \mathrm{q}=0.0044, \mathrm{CDK} 13^{\mathrm{R} 860 \mathrm{Q}} \mathrm{q}=0.0009$. Others non-

820 significant. Mean + SD. $n=3$ biologic replicates. G-H) anti-CDK13 human melanoma cell ChIP-

821 seq displayed as a G) metagene or $\mathrm{H}) \log 2$ from $E G F P$ vs. $C D K 13^{W 878 L}$ for each gene. I) anti-

822 RNAPII human melanoma cell ChIP-seq metagene. See also Figure S2. 
Figure 3
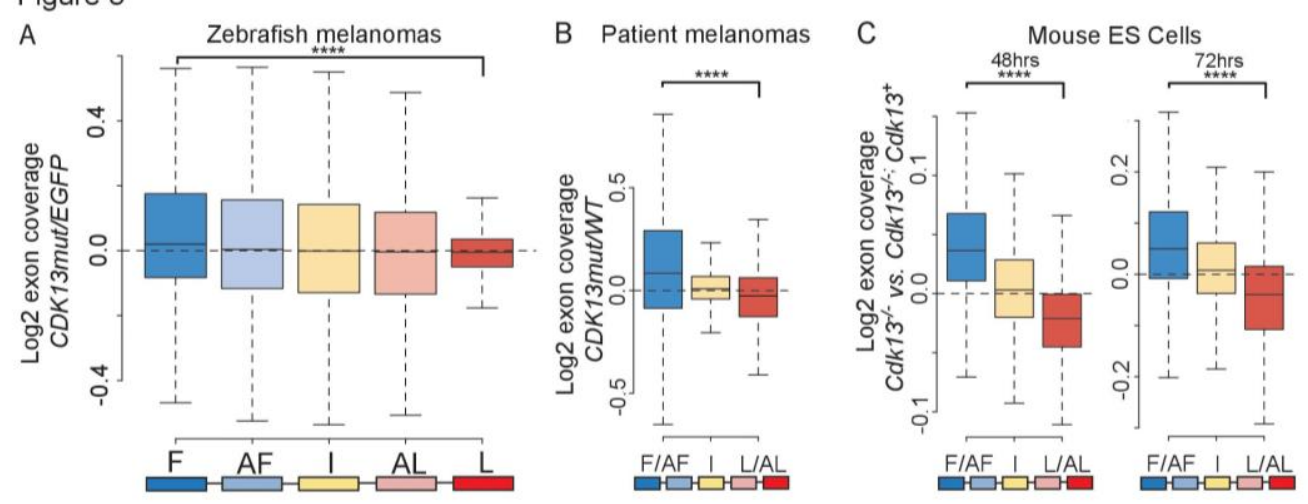

D

E Mouse ES Cells
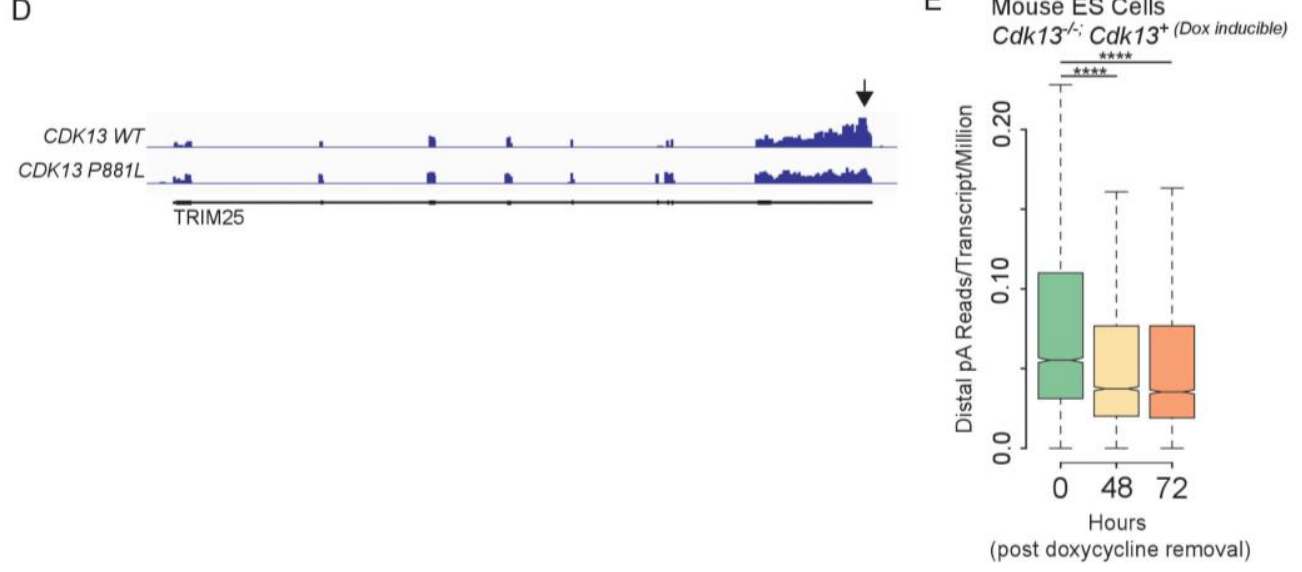

Figure 3: CDK13 mutation/depletion results in accumulation of prematurely terminated RNAs. A) Distributions of the $\log _{2}$-fold differences in normalized exon coverage for each exon category in $C D K 13 R 860 Q(\mathrm{n}=5)$ vs. EGFP $(\mathrm{n}=4)$ expressing zebrafish melanomas. $\mathrm{F}=$ first exon. $\mathrm{AF}=$ alternative first exon. $\mathrm{I}=$ internal exon. $\mathrm{AL}=$ alternative last exon. $\mathrm{L}=$ last exon. $* * * *=\mathrm{p}<2.2 \times 10-16, \mathrm{~F}$ vs. L exon. B) As in (A) plotted for $C D K 13$ mutant (n=3) vs. CDK13 WT

$831(\mathrm{n}=5)$ matched control patient melanomas. $* * * *=\mathrm{p}<2.2 \times 10-16, \mathrm{~F} / \mathrm{AF}$ vs. L/AL exon. C) As in

832 (A) plotted for $C d k 13-/-(\mathrm{n}=4)$ vs. $C d k 13-/-; C d k 13+$ mouse embryonic stem (ES) cells at 48

833 hours (left, $\mathrm{n}=4$ ) and 72 hours (right, $\mathrm{n}=4$ ). $* * * *=\mathrm{p}<2.2 \times 10-16, \mathrm{~F} / \mathrm{AF}$ vs. L/AL exon. D)

834 Example IGV track of melanoma patient RNA-seq for target gene TRIM25 from a patient with a $835 C D K 13^{P 881 L}$ mutation and a matched patient with CDK13 ${ }^{\mathrm{WT}}$. Arrow highlights 3 ' exon coverage

836 difference. E) Distribution of the ratios of reads representing distal (full-length) polyadenylation

837 site usage for each gene per transcript of that gene present in every million transcripts in the 838 sample (polyA reads/transcripts per million (TPM)) in Cdk13-/-; Cdk13+ mouse ES cells 0, 48, 839 and 72 hours after $\mathrm{Cdk} 13^{+}$depletion. $* * * *=\mathrm{p}<2.2 \times 10-16$. In all box plots, the black horizontal 
840 line indicates the median and whiskers extend to $1.5 \mathrm{x}$ the interquartile range. $\mathrm{p}$ values were 841 determined by two sided Wilcoxon rank sum test. See also Figure S3. 
Figure 4

A

B
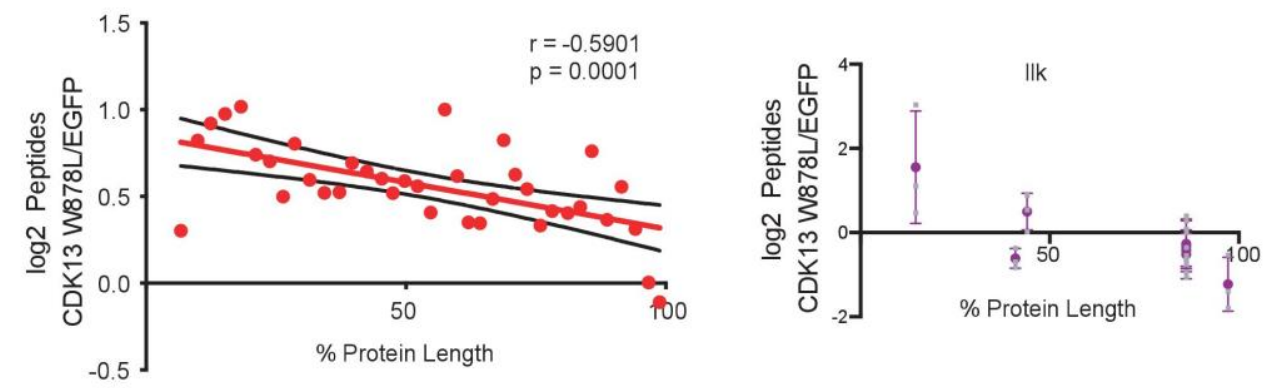

843

C
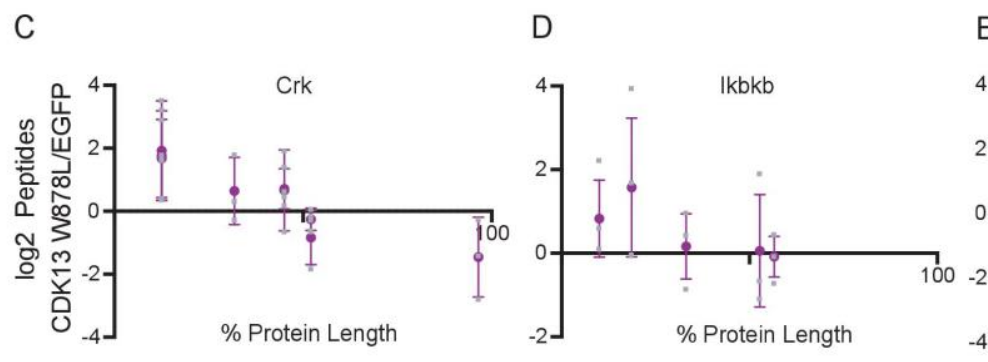

E

844

845 Figure 4: Mutant CDK13 caused accumulation of truncated proteins. A) Log2 peptide

846 measurement ratio between cells bearing CDK13 ${ }^{\mathrm{mel}}$ and EGFP plotted across a normalized

847 protein length. $\mathrm{r}=-0.5901, \mathrm{p}=0.001 . \mathrm{n}=3$ primary zebrafish melanomas from each condition.

848 B-E) Log2 CDK13 $13^{\text {el }} / E G F P$ zebrafish melanoma tandem mass spectrometry peptide

849 measurements plotted by \% protein length for candidate dominant negative protein targets B) Ilk,

850 C) Crk, D) Ikbkb, and E) Cdk15. Gray squares = individual measurements. Error bars = SD. n=3

851 zebrafish melanomas of each genotype. See also Figure S4. 
Figure 5

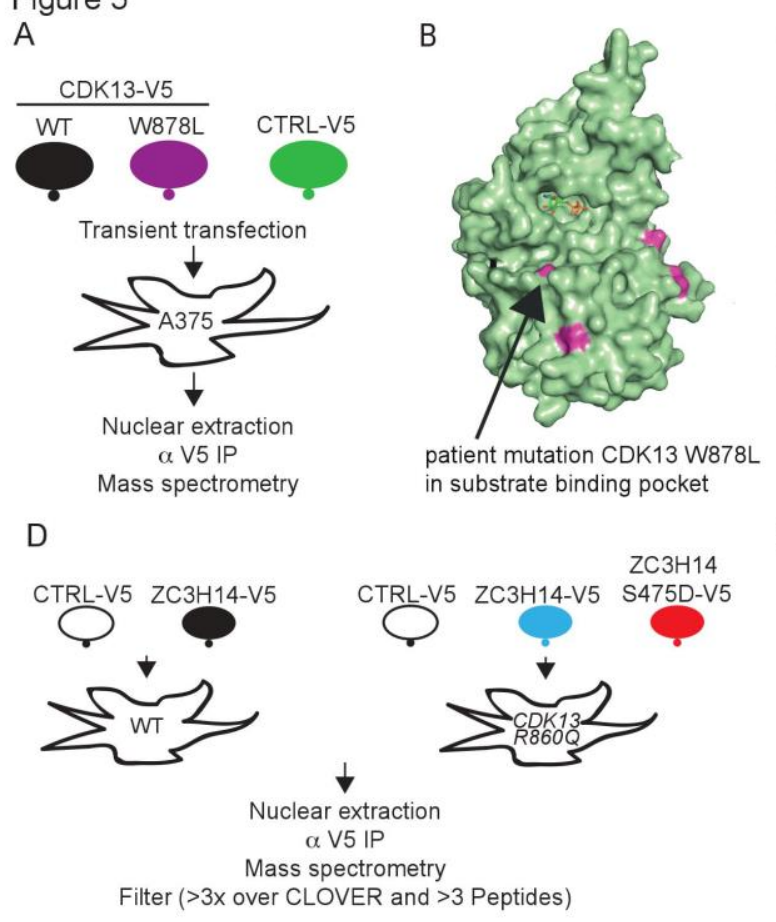

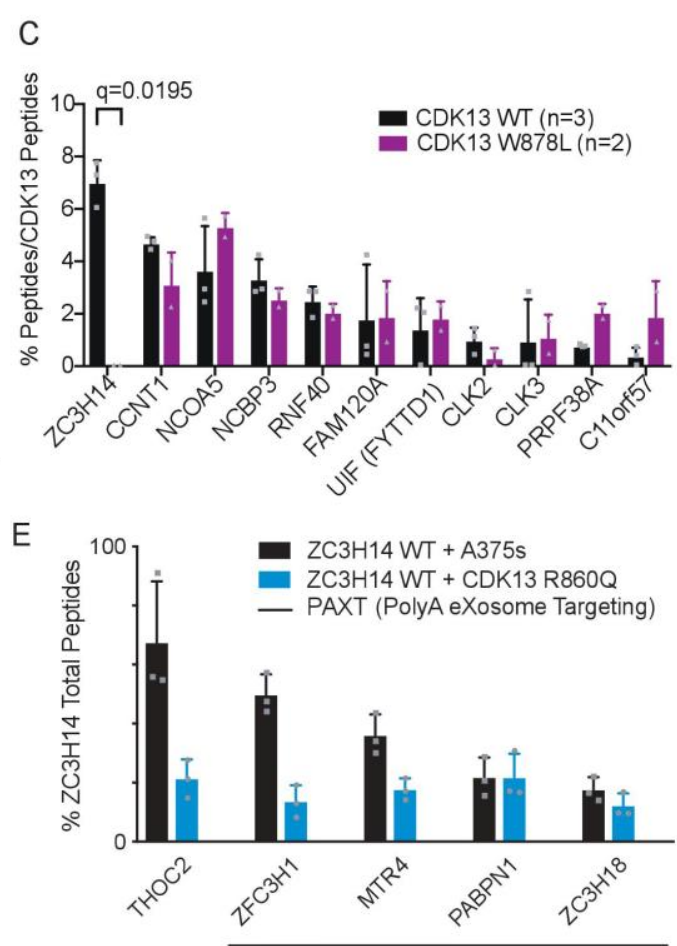

Figure 5: Mutant CDK13 disrupts the polyA RNA exosome.

856 A) Immunoprecipitation mass spectrometry (IP-MS) scheme. B) Surface display of W878L

857 patient mutation in substrate binding groove. C) IP-MS most abundant proteins isolated as a percent of CDK13 peptides isolated. $q=$ unpaired $t$ test with multiple test correction. Mean + SD. $\mathrm{n}=$ biologic replicates. Gray dots = individual values. D) ZC3H14 IP-MS scheme in CDK13 mutant expressing human melanoma cells. E) IP-MS of PAXT complex proteins isolated as a percent of $\mathrm{ZC} 3 \mathrm{H} 14$ peptides isolated. Mean $+\mathrm{SD}$. n=biologic replicates. Gray dots = individual values. See also Figure S5. 


\section{Extended Data}

Figure S1

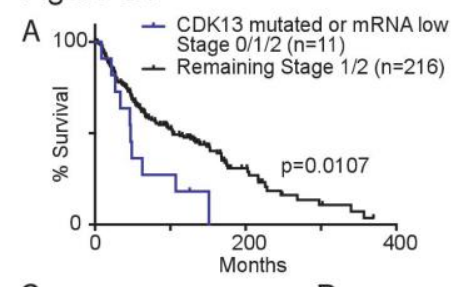

c

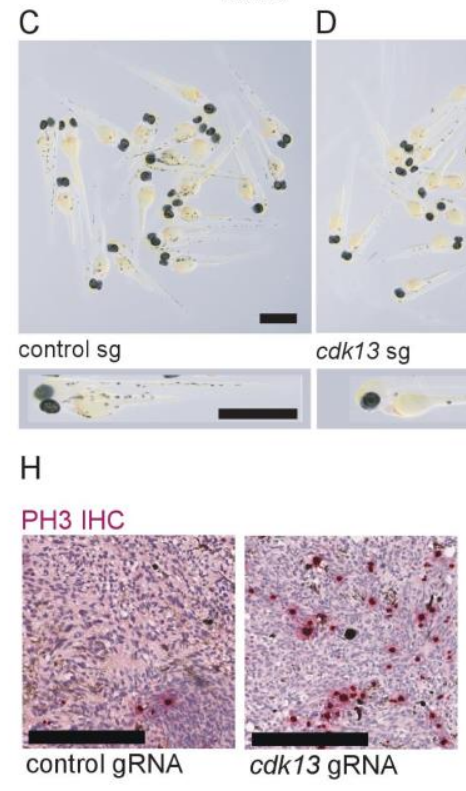

$\mathrm{K}$

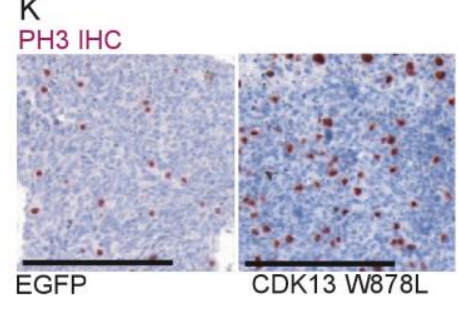

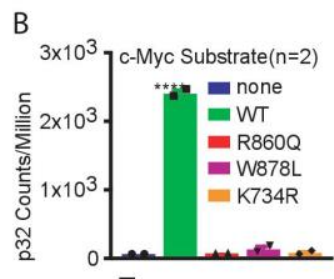

$\mathrm{E}$
$\mathrm{F}$

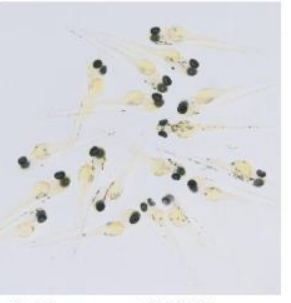

cdk13 sg +oeCDK13
G

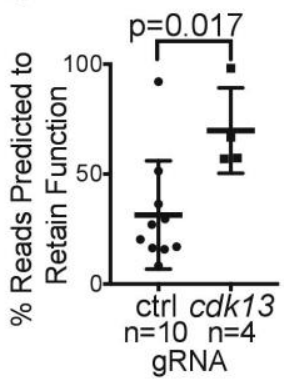

I

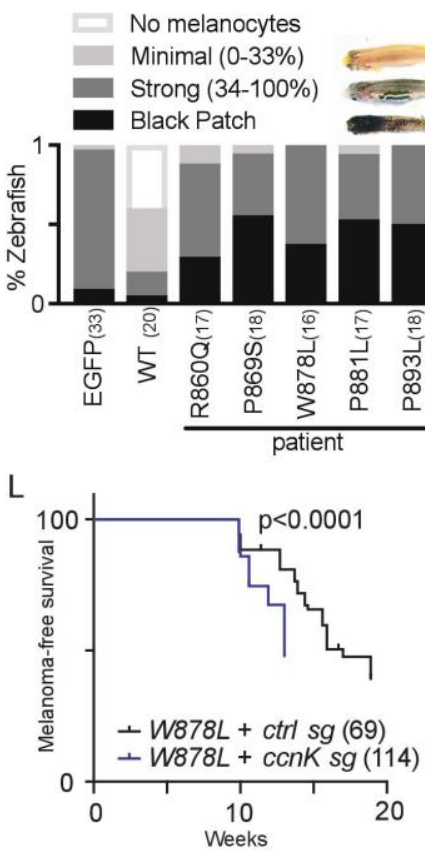

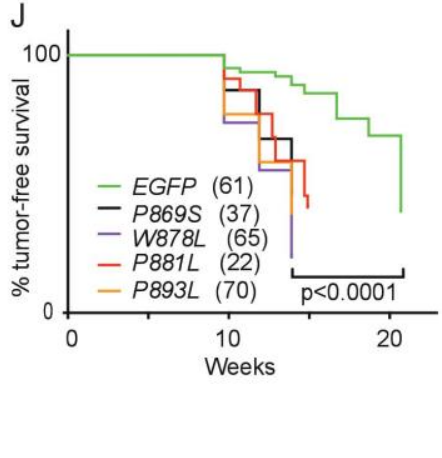

$\mathrm{M}$

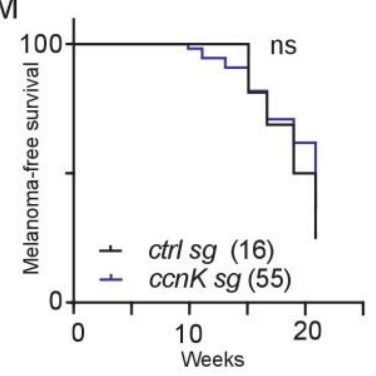

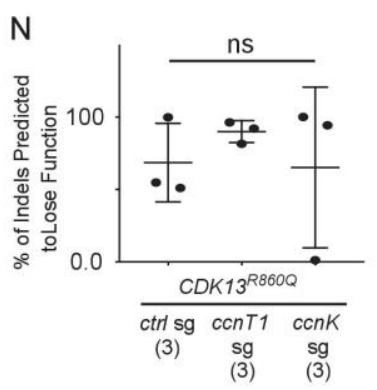

0

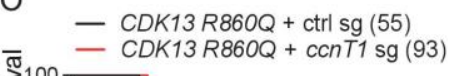

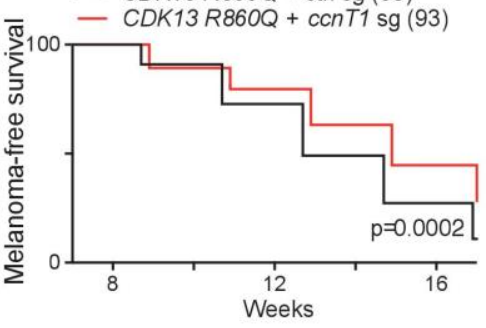

873 Figure S1: A) Downregulation or mutation of CDK13 in patients initially staged as 0/1/2 is 
874 associated with worse overall survival compared to remaining patients initially staged $1 / 2$.

$875 \mathrm{p}=0.0107$. Log-rank. $\mathrm{n}=$ patients. B) In vitro kinase assay using wild type and patient-mutated

876 CDK13 activated by CCNK on c-Myc substrate. One-way ANOVA with no kinase vs. all

877 conditions; WT CDK13 $* * * *=\mathrm{q}=0.0001$, all mutated CDK13 comparisons non-significant. Mean

$878+$-SD. $\mathrm{n}=2$ replicates. C-F) Light micrographs from 3 days post fertilization (dpf) mitfa:BRAF;

879 p53-/-; mitfa-/- zebrafish injected with C) control melanocyte specific CRISPR, D) $c d k 13$

880 melanocyte-specific CRISPR, E) melanocyte-specific overexpression of human $C D K 13^{W T}, \mathrm{~F}$ )

$881 c d k 13$ melanocyte-specific CRISPR and human $C D K 13^{W T}$. Full image and inset scale bar $=$

882 100um. G) Representative PH3 staining image from $c d k 13$ gRNA and control gRNA zebrafish

883 melanomas. Scale bar $=200 \mu \mathrm{m}$. H) Quantification of PCR reads across the CRISPR site predicted

884 to retain function of $c d k 13 . \mathrm{p}=0.017$ (t-test, two tailed). Mean +/- SD. $\mathrm{n}=$ melanomas. I)

885 Quantification of pigmentation patterns of 9 week old mitfa:BRAF; p53-/-; mitfa-/- zebrafish

886 from Figure $1 \mathrm{H}$ injected with melanocyte-specific expression vectors for $E G F P, C D K 13^{W T}$,

$887 C D K 13$ patient mutations, or control catalytically dead $C D K 13^{K 734 R}$. White=no melanocytes,

888 light gray $=$ minimal melanocytes $(0-33 \%$ length $)$, dark gray $=$ strong $(34-100 \%$ length $)$, black =

889 black patch. (\#)=zebrafish. J) Melanoma-free survival curves of EGFP and patient-mutant

890 CDK13 melanomas. $\mathrm{P}<0.0001$ (log rank). (\#)=zebrafish. K) Representative PH3 staining image

891 from $E G F P$ and $C D K 13^{W 878 L}$ expressing zebrafish melanomas. Scale bar=200 $\mu \mathrm{m}$. L) Melanoma-

892 free survival of melanocyte-specific expression of CDK13 W878L coinjected with melanocyte-

893 specific CRISPR of either a control gRNA or $c c n K$ gRNA. p<0.0001 (log-rank). (\#)=zebrafish.

894 M) Melanoma-free survival of melanocyte-specific expression of control gRNA or ccnK gRNA.

895 ns=non significant (log-rank). (\#)=zebrafish. N) \% CRISPR insertion/deletions (indels) predicted

896 to lose function in $C D K 13 R 860 Q+$ control gRNA, $C D K 13 R 860 Q+c c n T 1$ gRNA, and $C D K 13$

$897 R 860 Q+\operatorname{ccnK}$ gRNA melanomas. Unpaired t-test, two tailed, non-significant. Mean +/-SD.

898 (\#)=melanomas. O) Melanoma-free survival of mitfa:BRAF; p53-/-; mitfa-/- zebrafish with

899 melanocyte specific CDK13 R860Q expression and melanocyte-specific CRISPR of either

900 control gRNA or $c c n T 1$ gRNA. p=0.0002, log rank. (\#)=zebrafish. 
Figure S2
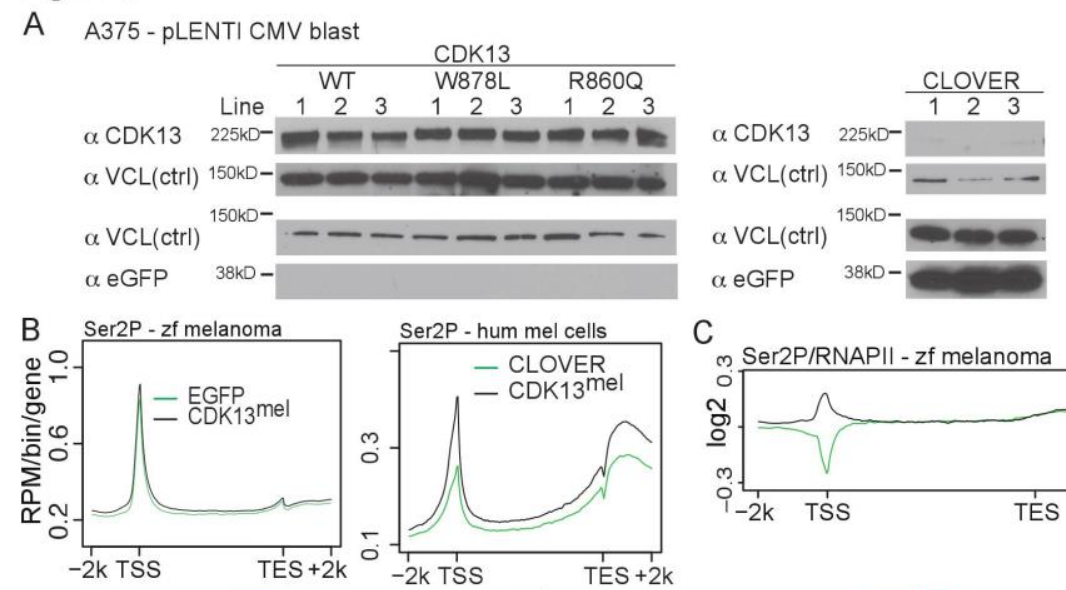

C

D
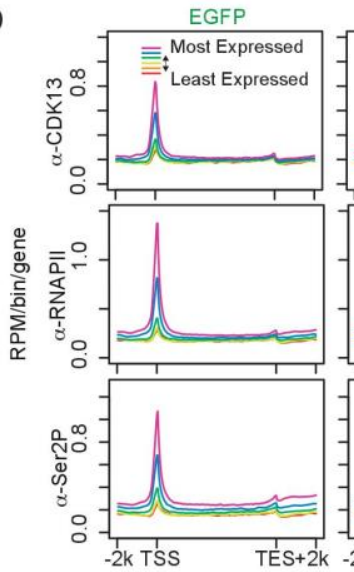

F
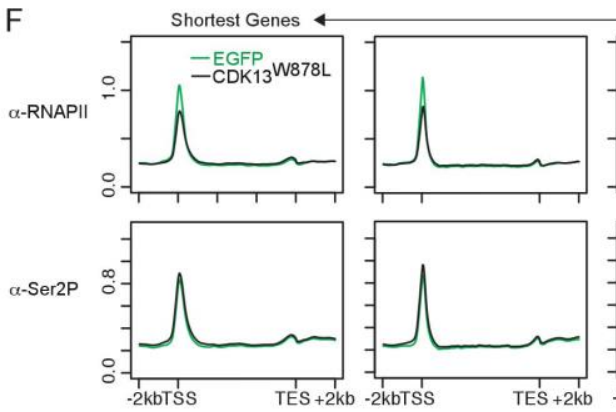

G

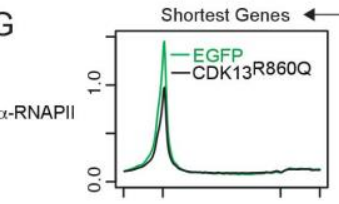

$\alpha$-Ser2P

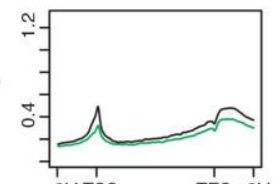

CDK13mel
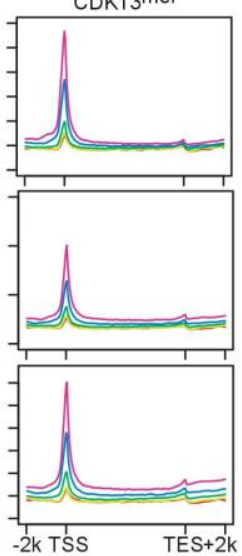
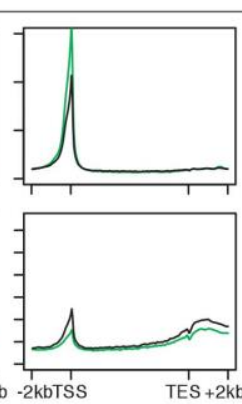

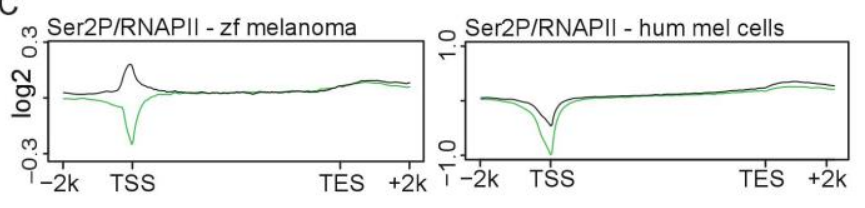

E
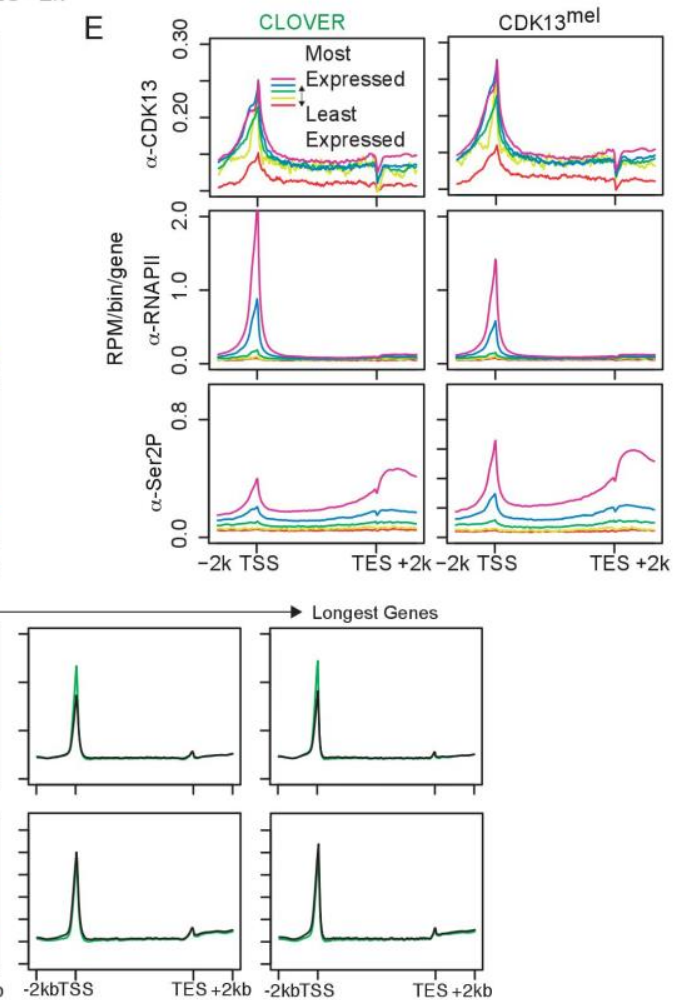

902
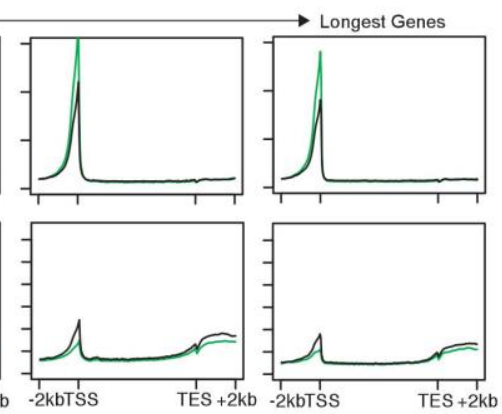

903 Figures S2: A) Immunoblot showing overexpression of CDK13 (WT, W878L, or R860Q) or

904 CLOVER control in three independently derived lines. B) anti-RNAPII Ser2p metagene in 
905 zebrafish melanoma (left) and in human melanoma cells (right). C) ChIP-seq metagenes of Ser2p

906 versus total RNAPII at genes with the largest fold increase in promoter CDK13 ${ }^{\text {mel }}$ (left) in

907 zebrafish melanoma and (right) in human melanoma cells. D-E) ChIP-seq metagenes for EGFP

908 (left) and CDK13 ${ }^{\text {mel }}$ (right) with anti-CDK13 (top), anti-8WG16 (middle), and anti-S2 CTD

909 (bottom) in expression group sextiles. Purple = most highly expressed. Red = most lowly

910 expressed for D) zebrafish melanomas and E) human melanoma cells. F-G) ChIP-seq metagenes

911 for control (green) and mutant CDK13 (black) in gene length group sextiles with anti-RNAPII

912 (top), and anti-S2 CTD (bottom) from F) zebrafish melanomas and in G) human melanoma cells.

913 Longest and shortest gene groups removed for viewing ease. TSS = transcriptional start site. TES

$914=$ transcriptional end site. $2 \mathrm{k}=2 \mathrm{kilobases} . \mathrm{RPM}=$ reads per million. 
Figure S3

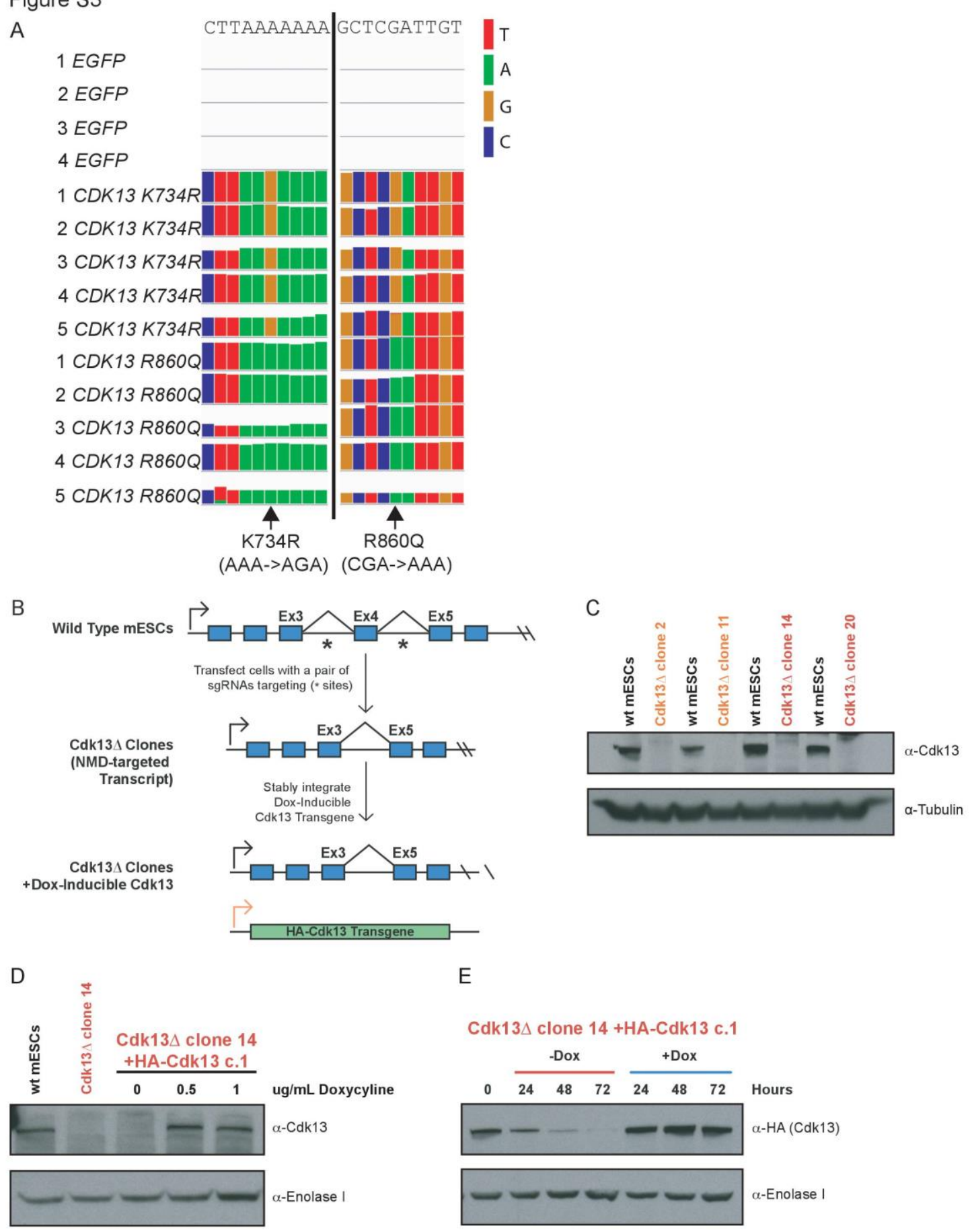

917 Figure S3: A) IGV track from zebrafish melanoma RNA-seq bam files confirming CDK13

918 mutant overexpression. Arrows=mutant base pairs. B) Schematic for generation of Cdk13-/- 
919 mouse embryonic stem cells (mESCs) with doxycycline-inducible Cdk13-HA for

920 complementation. C) Representative immunoblot of $n=4$ showing $4 \mathrm{mESC}$ clones with loss of

921 Cdk13. Orange $=$ gRNA pair 1 . Red=gRNA pair 2. D) Representative immunoblot of $\mathrm{n}=2$

922 (independently derived clones) showing near wild type level of rescue upon expression of

923 Cdk13-HA upon expression of doxycycline. F) Representative immunoblot of $\mathrm{n}=2$

924 (independently derived clones) of $C d k 13-H A$ upon doxycycline withdrawal. RNA was gathered 925 at 48 and 72 hours. 
Figure S4

A

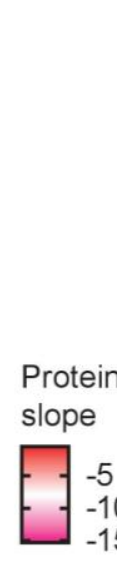

Figure S4: A) Heatmap of proteins with evidence of truncation in zebrafish CDK13 ${ }^{\text {mel }}$ as compared with EGFP melanomas. Left $=$ heatmap of $\log 2$ fold change slope measurements. Right $=$

$F$ value heatmap of $F$ value (degree of significance, all included genes had $\mathrm{F}$ value indicating $\mathrm{p}<0.05)$. 
Figure S5

A

\begin{tabular}{|c|c|c|c|c|c|}
\hline \multirow{2}{*}{$\begin{array}{l}\text { ZC3H14 } \\
\begin{array}{|l|l|}\text { PW-like } \\
\end{array}\end{array}$} & \multicolumn{4}{|r|}{ NLS } & \multirow[b]{2}{*}{$(\mathrm{CCCH}) 2$} \\
\hline & Q-rich & il & & & \\
\hline 96 & & $292-308$ & & $170-473$ & $601-716 \quad 736$ \\
\hline & & $\begin{array}{c}\text { S343 } \\
\text { P }\end{array}$ & $\begin{array}{c}S 409 \\
\mathrm{P}\end{array}$ & $\begin{array}{c}\text { S475 S515 } \\
\text { P P }\end{array}$ & \\
\hline$Q$ & & $\mathrm{P}$ & $\mathrm{P}$ & $x$ & \\
\hline
\end{tabular}

B

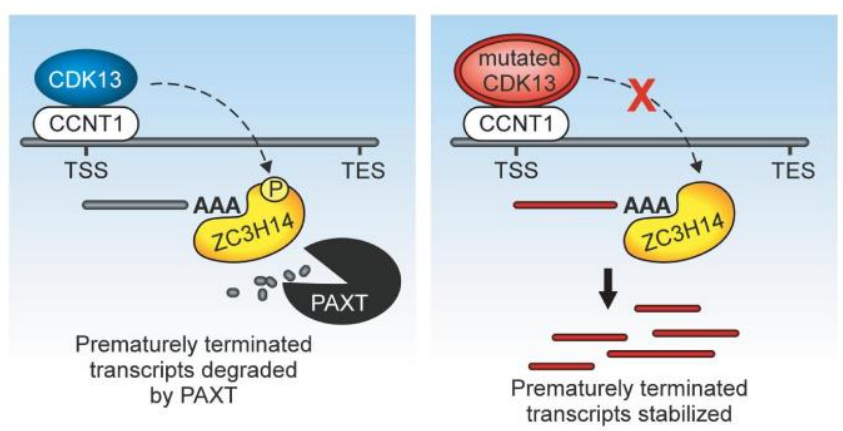

958

959 Figure S5: A) Graphic depicting ZC3H14 phosphorylations identified with in all three replicates

960 of human melanoma cells with CDK13 ${ }^{\mathrm{WT}}$ or CDK13 ${ }^{\mathrm{R} 860 \mathrm{Q}}$ expression. B) Model of CDK13's

961 activation of PAXT.

962

\section{References (including methods references):}

964 1. Malumbres, M. Cyclin-dependent kinases. 15, 1-10 (2014).

965 2. Schwartz, B. E., Larochelle, S., Suter, B. \& Lis, J. T. Cdk7 is required for full activation

966 of Drosophila heat shock genes and RNA polymerase II phosphorylation in vivo. Mol.

$967 \quad$ Cell. Biol. 23, 6876-6886 (2003).

968 3. McCracken, S. et al. 5'-Capping enzymes are targeted to pre-mRNA by binding to the phosphorylated carboxy-terminal domain of RNA polymerase II. Genes Dev. 11, 33063318 (1997).

4. Shim, E. Y., Walker, A. K., Shi, Y. \& Blackwell, T. K. CDK-9/cyclin T (P-TEFb) is required in two postinitiation pathways for transcription in the C. elegans embryo. Genes Dev. 16, 2135-2146 (2002).

5. Adelman, K. \& Lis, J. T. Promoter-proximal pausing of RNA polymerase II: emerging roles in metazoans. Nature Reviews Genetics 13, 720-731 (2012).

6. Wang, Y. et al. CDK7-Dependent Transcriptional Addiction in Triple-Negative Breast Cancer. Cell 163, 174-186 (2015).

7. Christensen, C. L. et al. Targeting Transcriptional Addictions in Small Cell Lung Cancer with a Covalent CDK7 Inhibitor. Cancer Cell 26, 909-922 (2014).

8. Chipumuro, E. et al. CDK7 Inhibition Suppresses Super-Enhancer-Linked Oncogenic Transcription in MYCN-Driven Cancer. Cell 159, 1126-1139 (2014).

9. Kwiatkowski, N. et al. Targeting transcription regulation in cancer with a covalent CDK7 inhibitor. Nature 511, 616-620 (2014). 
984 10. Deciphering Developmental Disorders Study. Prevalence and architecture of de novo

985

986

987

988

989

990

991

992

993

994

995

996

997

998

999

1000

1001

1002

1003

1004

1005

1006

1007

1008

1009

1010

1011

1012

1013

1014

1015

1016

1017

1018

1019

1020

1021

1022

1023

1024

1025

1026

1027

1028

1029

mutations in developmental disorders. Nature 542, 433-438 (2017).

11. Sifrim, A. et al. Distinct genetic architectures for syndromic and nonsyndromic congenital heart defects identified by exome sequencing. Nature Genetics 48, 1060-1065 (2016).

12. Gu, B., Eick, D. \& Bensaude, O. CTD serine-2 plays a critical role in splicing and termination factor recruitment to RNA polymerase II in vivo. Nucleic Acids Res 41, 15911603 (2012).

13. Lubas, M. et al. Interaction Profiling Identifies the Human Nuclear Exosome Targeting Complex. Mol. Cell 43, 624-637 (2011).

14. Meola, N. et al. Identification of a Nuclear Exosome Decay Pathway for Processed Transcripts. Mol. Cell 64, 520-533 (2016).

15. Ogami, K. et al. An Mtr4/ZFC3H1 complex facilitates turnover of unstable nuclear RNAs to prevent their cytoplasmic transport and global translational repression. Genes Dev. 31, 1257-1271 (2017).

16. Lawrence, M. et al. Software for computing and annotating genomic ranges. PLoS Comput. Biol. 9, e1003118 (2013).

17. Cerami, E. et al. The cBio cancer genomics portal: an open platform for exploring multidimensional cancer genomics data. Cancer Discovery 2, 401-404 (2012).

18. Greifenberg, A. K. et al. Structural and Functional Analysis of the Cdk13/Cyclin K Complex. Cell Reports 14, 320-331 (2016).

19. Kaufman, C. K. et al. A zebrafish melanoma model reveals emergence of neural crest identity during melanoma initiation. Science 351, aad2197 (2016).

20. Patton, E. E. et al. BRAF Mutations Are Sufficient to Promote Nevi Formation and Cooperate with p53 in the Genesis of Melanoma. Current Biology 15, 249-254 (2005).

21. Ceol, C. J. et al. The histone methyltransferase SETDB1 is recurrently amplified in melanoma and accelerates its onset. Nature 471, 513-517 (2011).

22. Liang, K. et al. Characterization of human cyclin-dependent kinase 12 (CDK12) and CDK13 complexes in C-terminal domain phosphorylation, gene transcription, and RNA processing. Mol. Cell. Biol. 35, 928-938 (2015).

23. Blazek, D. et al. The Cyclin K/Cdk12 complex maintains genomic stability via regulation of expression of DNA damage response genes. Genes Dev. 25, 2158-2172 (2011).

24. Even, Y., Escande, M.-L., Fayet, C. \& Genevière, A.-M. CDK13, a Kinase Involved in Pre-mRNA Splicing, Is a Component of the Perinucleolar Compartment. PLoS ONE 11, e0149184 (2016).

25. Davidson, L., Muniz, L. \& West, S. 3' end formation of pre-mRNA and phosphorylation of Ser2 on the RNA polymerase II CTD are reciprocally coupled in human cells. Genes Dev. 28, 342-356 (2014).

26. Buratowski, S. Progression through the RNA Polymerase II CTD Cycle. Mol. Cell 36, 541-546 (2009).

27. Fong, N., Saldi, T., Sheridan, R. M., Cortazar, M. A. \& Bentley, D. L. RNA Pol II Dynamics Modulate Co-transcriptional Chromatin Modification, CTD Phosphorylation, and Transcriptional Direction. Mol. Cell 66, 546-557.e3 (2017).

28. Dubbury, S. J., Boutz, P. L. \& Sharp, P. A. CDK12 regulates DNA repair genes by suppressing intronic polyadenylation. Nature 564, 141-145 (2018).

29. Reimer, M., Denby, E., Zustiak, S. P. \& Schober, J. M. Ras GAP-related and C-terminal domain-dependent localization and tumorigenic activities of IQGAP1 in melanoma cells. 
PLoS ONE 12, e0189589-16 (2017).

1031

1032

1033

1034

1035

1036

1037

1038

1039

1040

1041

1042

1043

1044

1045

1046

1047

1048

1049

1050

1051

1052

1053

1054

1055

1056

1057

1058

1059

1060

1061

1062

1063

1064

1065

1066

1067

1068

1069

1070

1071

1072

1073

1074

1075

30. Khyrul, W. A. K. M., LaLonde, D. P., Brown, M. C., Levinson, H. \& Turner, C. E. The Integrin-linked Kinase Regulates Cell Morphology and Motility in a Rho-associated Kinase-dependent Manner. J. Biol. Chem. 279, 54131-54139 (2004).

31. May, M. J., Marienfeld, R. B. \& Ghosh, S. Characterization of the IkB-kinase NEMO Binding Domain. J. Biol. Chem. 277, 45992-46000 (2002).

32. The Gene Ontology Consortium. The Gene Ontology Resource: 20 years and still GOing strong. Nucleic Acids Res 47, D330-D338 (2018).

33. Ashburner, M. et al. Gene Ontology: tool for the unification of biology. Nature Genetics 25, 25-29 (2000).

34. Dai, Q. et al. Cyclin K-containing Kinase Complexes Maintain Self-renewal in Murine Embryonic Stem Cells. J. Biol. Chem. 287, 25344-25352 (2012).

35. Rha, J. et al. The RNA-binding protein, $\mathrm{ZC} 3 \mathrm{H} 14$, is required for proper poly(A) tail length control, expression of synaptic proteins, and brain function in mice. Human Molecular Genetics 26, 3663-3681 (2017).

36. Pak, C. et al. Mutation of the conserved polyadenosine RNA binding protein, ZC3H14/dNab2, impairs neural function in Drosophila and humans. Proc Natl Acad Sci $U$ $S$ A 108, 12390-12395 (2011).

37. Pak, C. et al. Mutation of the conserved polyadenosine RNA binding protein, ZC3H14/dNab2, impairs neural function in Drosophila and humans. Proc Natl Acad Sci $U$ $S$ A 108, 12390-12395 (2011).

38. Lee, S.-H. et al. Widespread intronic polyadenylation inactivates tumour suppressor genes in leukaemia. Nature 561, 127-131 (2018).

39. Krajewska, M. et al. CDK12 loss in cancer cells affects DNA damage response genes through premature cleavage and polyadenylation. Nature Communications 10, 1757-1773 (2019).

40. Akbani, R. et al. Genomic Classification of Cutaneous Melanoma. Cell 161, 1681-1696 (2015).

41. Montague, T. G., Cruz, J. M., Gagnon, J. A., Church, G. M. \& Valen, E. CHOPCHOP: a CRISPR/Cas9 and TALEN web tool for genome editing. Nucleic Acids Res 42, W401W407 (2014).

42. Labun, K., Montague, T. G., Gagnon, J. A., Thyme, S. B. \& Valen, E. CHOPCHOP v2: a web tool for the next generation of CRISPR genome engineering. Nucleic Acids Res 44, W272-6 (2016).

43. Meeker, N. D., Hutchinson, S. A., Ho, L. \& Trede, N. S. Method for isolation of PCRready genomic DNA from zebrafish tissues. BioTechniques 43, 610-614 (2007).

44. Campeau, E. et al. A Versatile Viral System for Expression and Depletion of Proteins in Mammalian Cells. PLoS ONE 4, e6529-18 (2009).

45. Lee, T. I., Johnstone, S. E. \& Young, R. A. Chromatin immunoprecipitation and microarray-based analysis of protein location. Nat Protoc 1, 729-748 (2006).

46. Langmead, B., Trapnell, C., Pop, M. \& Salzberg, S. L. Ultrafast and memory-efficient alignment of short DNA sequences to the human genome. Genome Biol. 10, R25 (2009).

47. Zhang, Y. et al. Model-based analysis of ChIP-Seq (MACS). Genome Biol. 9, R137 (2008).

48. Thorvaldsdóttir, H., Robinson, J. T. \& Mesirov, J. P. Integrative Genomics Viewer (IGV): high-performance genomics data visualization and exploration. Brief. Bioinformatics 14, 
1076

1077

1078

1079

1080

1081

1082

1083

1084

1085

1086

1087

1088

1089

1090

1091

1092

1093

1094

1095

1096

1097

1098

1099

1100

1101

1102

1103

1104

1105

1106

1107

1108

1109

178-192 (2013).

49. Robinson, J. T. et al. Integrative genomics viewer. Nature Publishing Group 29, 24-26 (2011).

50. Lawrence, M. et al. Software for computing and annotating genomic ranges. PLoS Comput. Biol. 9, e1003118 (2013).

51. Lawrence, M., Gentleman, R. \& Carey, V. rtracklayer: an R package for interfacing with genome browsers. Bioinformatics 25, 1841-1842 (2009).

52. Kim, D. et al. TopHat2: accurate alignment of transcriptomes in the presence of insertions, deletions and gene fusions. Genome Biol. 14, R36 (2013).

53. Anders, S., Pyl, P. T. \& Huber, W. HTSeq--a Python framework to work with highthroughput sequencing data. Bioinformatics 31, 166-169 (2015).

54. Love, M. I., Huber, W. \& Anders, S. Moderated estimation of fold change and dispersion for RNA-seq data with DESeq2. Genome Biol. 15, 31-21 (2014).

55. Ran, F. A. et al. Genome engineering using the CRISPR-Cas9 system. Nat Protoc 8, 2281-2308 (2013).

56. Cong, L. et al. Multiplex genome engineering using CRISPR/Cas systems. Science 339, 819-823 (2013).

57. Li, H. et al. The Sequence Alignment/Map format and SAMtools. Bioinformatics 25, 2078-2079 (2009).

58. Anders, S., Reyes, A. \& Huber, W. Detecting differential usage of exons from RNA-seq data. Genome Res. 22, 2008-2017 (2012).

59. Reyes, A. et al. Drift and conservation of differential exon usage across tissues in primate species. Proc Natl Acad Sci U S A 110, 15377-15382 (2013).

60. Viswanathan, S. R. et al. Structural Alterations Driving Castration-Resistant Prostate Cancer Revealed by Linked-Read Genome Sequencing. Cell 174, 433-447.e19 (2018).

61. Bell, D. et al. Integrated genomic analyses of ovarian carcinoma. Nature 474, 609-615 (2011).

62. Hamilton, M. J. et al. Heterozygous mutations affecting the protein kinase domain of CDK13cause a syndromic form of developmental delay and intellectual disability. $J$ Med Genet 55, 28-38 (2017).

63. Ekumi, K. M. et al. Ovarian carcinoma CDK12 mutations misregulate expression of DNA repair genes via deficient formation and function of the Cdk12/CycK complex. Nucleic Acids Res 43, 2575-2589 (2015). 